\title{
LLECMOD: A Bivariate Population Balance Simulation Tool for Pulsed Liquid-Liquid Extraction Columns
}

\author{
Moutasem M. Jaradat ${ }^{\mathrm{a}, \mathrm{c}}$, Menwer M. Attarakih ${ }^{\mathrm{a}, \mathrm{b}}$, Tilmann Steinmetz ${ }^{\mathrm{d}}$ and Hans-Jörg Bart*,a,c \\ ${ }^{a}$ Chair of Separation Science and Technology, TU Kaiserslautern, P.O. Box 3049, 67653 Kaiserslautern, Germany \\ ${ }^{b}$ Chem. Eng. Department, Faculty of Engineering \& Technology, University of Jordan, 11942 Amman, Jordan \\ ${ }^{c}$ Centre of Mathematical and Computational Modeling, TU Kaiserslautern \\ ${ }^{d}$ BASF SE, 67056 Ludwigshafen, Germany
}

\begin{abstract}
A rigours mathematical model based on the bivariate population balance frame work (the base of LLECMOD "Liquid-Liquid Extraction Column Module") for the steady state and dynamic simulation of pulsed liquid-liquid extraction columns is developed. The model simulates the coupled hydrodynamic and mass transfer for pulsed (packed and sieve plate) extraction columns. It is implemented using visual digital FORTRAN and then integrated into the LLECMOD program. Experimental validated correlations are used for the estimation of the droplet terminal velocity in extraction columns based on single and swarm droplet experiments in laboratory scale devices. Additionally, recently published correlations for turbulent energy dissipation, droplet breakage and coalescence frequencies are discussed as being used in this version of LLECMOD. In a case study, LLECMOD is used here to simulate the steady state performance of pulsed extraction columns with two chemical test systems recommended by the European Federation of Chemical Engineering (water-acetone-n-butyl acetate and water-acetone-toluene) and an industrial test system. Model predictions are successfully validated against steady state and transient experimental data, where good agreements are achieved. The simulated results (holdup, mean droplet diameter and mass transfer profiles) compared to the experimental data show that LLECMOD is a powerful simulation tool, which can efficiently predict the dynamic and steady state performance of pulsed extraction columns.
\end{abstract}

Keywords: LLECMOD, population balance modeling, simulation, breakage, coalescence, mass transfer, hydrodynamics, transient analysis, pulsed sieve plate extraction column, pulsed packed extraction column.

\section{INTRODUCTION}

Liquid-liquid extraction is an important separation process encountered in numerous chemical process industries $[1,2]$. Very often it is performed in column extractors offering high throughputs with small footprint. Different types of liquid-liquid extraction columns are being used in chemical industries, which can be classified into two main categories: stirred and pulsed columns. The latter ones (with/without pulsation) are packed and sieve plate columns. Pulsed columns have the advantage of easy maintenance as all moving parts (pulsation equipment) are located outside the column shell. This pulsing action reduces droplet size of the dispersed phase independently of flow rates and improves the mass transfer. The most common type of pulsed columns, which particularly used in the nuclear industry is the pulsed sieve plate column. Packed columns are packed with commercially available (random or structured) packing materials, which ensure that the two phases are in intensive contact. Packed columns have no moving parts and are relatively simple to operate and best to use at low interfacial tension.

*Address correspondence to this author at the Chair of Separation Science and Technology, TU Kaiserslautern, P.O. Box 3049, 67653 Kaiserslautern, Germany; Tel: +49631-205-2414; Fax: +49-631-205-2119;

E-mail: bart@mv.uni-kl.de
To shed more light on the extraction behavior in the pulsed packed and sieve plate columns, the hydrodynamics as well as the mass transfer characteristics must be well understood. Our present knowledge of the design and performance of extraction columns is still far from satisfactory. The reason is mainly due to the complex behavior of the hydrodynamics and mass transfer [3]. It is obvious that the changes in the characteristics (interfacial tension, Sauter diameter, etc.) of the droplet population along the column have to be considered in order to describe conveniently the behavior of the column. The dispersed phase in the case of liquidliquid extraction undergoes formidable changes and loses its identity continuously as the droplets break and coalesce. Accordingly, detailed modeling on a discrete level is needed using the population balance equation as a mathematical framework. The multivariate non-equilibrium population balance models have emerged as an effective tool for the study of the complex coupled hydrodynamics and mass transfer in liquid-liquid extraction columns [4]. Unfortunately, the population balance modeling of extraction columns results in a set of integro-partial differential equations with no general analytical solution. Additionally, even the numerical solution of PBEs is still not efficiently developed, particularly when coupled hydrodynamics and mass transfer has to be considered. The main objective of this work is to 
develop a model that is capable to describe the dynamic and steady state behavior of pulsed packed and sieve tray extraction columns. The models of both columns are integrated into the existing program: LLECMOD $[5,6]$, which can also simulate agitated extraction columns (RDC and Kühni). LLECMOD simulates steady state and dynamic behavior and takes into account the effect of dispersed phase inlet (light or heavy phase is dispersed) and the direction of mass transfer (from continuous to dispersed phase and vice versa) [7]. Therefore, scale-up studies and performance simulations of agitated and non-agitated extraction columns based on population balance modeling can thus be carried out successfully.

\section{THE MATHEMATICAL MODEL}

Simulating liquid-liquid extraction columns is a challenging task due to the discrete character of the dispersed phase. This is resulted from random breakage and coalescence of droplets processes, which are highly coupled to the mass transport of solutes between the two counter-flowing phases. Modeling of such complex transport phenomena is resolved by using a multivariate population balance equation. The population of droplets is modelled by a multivariate number concentration function, which takes into account the dynamic evolution of droplet size and solute concentrations. The understanding of liquid-liquid extraction columns dynamic behavior can be notably used in the design of process control strategy or the start-up and shutdown procedures [811]. In attempting to model this dynamic behavior, one has to consider the macroscopic dispersed phase interactions (droplet breakage and coalescence) as well as the microscopic interaction (interphase mass transfer) occurring in the continuously turbulent flow field, which results in a distributed population of droplets. This population is distributed not only in the spatial domain of the contacting equipment, but also randomly distributed with respect to the droplet properties such as size, concentration and age. Several attempts have been done to propose the proper dynamic model for liquid-liquid extraction columns [10-15]. The pseudohomogeneous models are too simple (by ignoring the discrete nature of the dispersed phase) to describe the particulate nature, where one of the liquid phases is normally dispersed as droplets in the second continuous phase [16]. Therefore, the influences of droplet movement, droplet interaction (breakage and coalescence), energy input (stirrer, pulsation) and mass transfer cannot be described satisfactorily. Several population balance models have been proposed by various authors. Garg and Pratt [17] have developed a population balance model for a pulsed sieve-plate extraction taking into account experimentally determined values for droplet breakage and coalescence. Casamatta and Vogelpohl [18] proposed a population balance model; Al Khani et al., [19] have applied this model for transient and steady state simulations of a pulsed sieve-plate extraction column. Milot et al. [20] have used the same basic model. A good review of which is presented by Gourdon et al., [21]. Recently, much work has been done using the population balance modeling in extraction columns [4-6, 21-25].

\subsection{Population Balance Model}

In modeling liquid extraction columns, the dispersed phase should not be considered as a quasi-continuous fluid, which is contradictory to the poly-dispersed character of the drops. Due to the presence of the breakage and coalescence mechanisms, relatively wide droplet size distributions often exist in extraction columns. In the population balance model, the droplets can be distinguished by their sizes, which are parameterized over the diameter or volume, under the assumption that all the droplets are spherical. Accordingly, population balance modeling gives the possibilities to improve the simulation quality to optimize the operation of extraction equipment $[4,24,26-29]$. The following integropartial differential equation represents the population balance equation (PBE):

$\frac{\partial n(v, \xi)}{\partial t}+\Delta \cdot\left[V_{d}-D_{d} \nabla n(v, \xi)\right]=\rho\{n(v, \xi), v\}$

In the above equation $n(t, d)$ is the number of droplets having sizes between $d$ and $d+\delta d$ per unit volume and $v$ is droplet volume, $D_{d}$ is axial dispersion coefficient, $V_{d}(z)$ is droplet velocity and $\rho(z)$ is the population balance source term, which takes into account droplet breakage and coalescence. The term in square brackets represents the transport either by convection, $V_{d}$ or fluctuation, $D_{d}$. The resulting equation determines the number of distribution of the droplets that can be classified based on their structure in the group of the Fokker-Planck equations. The Fokker-Planck equation is a pure differential equation and also known as generalized diffusion equation, Smoluchowski's equation or second Kolmogorov equation. The treatment and solution of this particular group of differential equations is described in detail in specialized literature. For example, one of the solution methods is the Monte Carlo method [30] or the extended fixed-pivot technique used in LLECMOD $[4,6]$. Further reading on the solution of the Fokker-Planck (population balance) equation can be found in literature $[6,23,29]$. The term $\rho\{n(v, \xi), v\}$ represents the instantaneous fluctuations in the population concentration due to droplet breakage and coalescence. In this term sources and sinks are considered by droplet breakage $\left(S_{B}\right)$, coalescence $\left(S_{C}\right)$ and the disperse phase inflow $\left(S_{F}\right)$ :

$\rho\{n(v, \xi), v\}=S_{F}+S_{B}+S_{C}$

This source term is described in detail by Attarakih et al., [6] and is summarized below for the purpose of clear presentation of the population balance model in LLECMOD. The inflow of disperse phase is considered as a point source term at the level $z_{d}$ using the Dirac delta function $\delta$. Thus the equation for the source term $S_{F}$ is written as:

$S_{F}=\frac{Q_{d}}{A} n_{F}\left(\frac{v(d)}{\bar{v}}\right) \delta(z-z d)$

Whereas:

$d_{\max }$ $\int_{0}^{\max } n_{F}(d) d=1$ 
where $n_{F}$ is the normalized number distribution density function for the inlet droplets. Whereas the source term for brekage $S_{B}$ and for coalescence $S_{F}$ are given in details by Attarakih et al., [6].

\subsection{Solution of the Population Balance Equation Using LLECMOD Program}

To solve the PBE in the simulation program LLECMOD $[5,6]$, the fixed-pivot technique [34] is extended to take into account solute transfer and external coordinate (column height) $[5,6]$. In this way, the bivariate PBE with respect to droplet size and concentration is decoupled into two population balances: One takes into account the droplet hydrodynamics and the other one takes into account solute transfer. This decoupling idea is based on using the Quadrature Method of Moments (QMOM) (see Attarakih et al., [4] for the details). The resulting system of PBEs is an integropartial differential system for which no general analytical solution is known. For this reason, these equations are cast into a system of hyperbolic conservation laws with integral source term with droplet diameter and column height as two independent coordinates. Concerning the droplet diameter, the population of droplets is categorized into a set of independent particles to statistically represent the numerous number of droplets flowing inside the column. Primary particles with fixed sizes are used to sample the number concentration function with local properties such as droplet holdup and solute concentration. This is done by the help of the fixed-pivot technique, which uses a discrete decomposition of the droplet volume $v$ into a finite number of grid points (primary particles) of size $x_{i}$. This allows two of the major populations which mean properties (droplet volume and solute concentrations) to be tracked directly using a fixed particle sizes. A detailed description of the solution algorithms is presented in Attarakih et al., [4,6]. The underlying idea begins by dividing the continuous droplet size into $M_{x}$ finite number of particles for which the ith-division is the local droplet holdup:

$$
\phi_{i}(t, \xi)=\int_{v_{i-1 / 2}}^{v_{i+1 / 2}} V_{d}(v) \cdot n(v, t, \xi) \delta v=V_{d}\left(x_{i}\right) N_{i}(t, \xi) \quad i \in\left[1, M_{x}\right]
$$

The global holdup of the dispersed phase can be calculated as: $\varphi=\sum_{i=1}^{M_{x}} \phi_{i}$. Thus, the integro-partial differential equations are replaced by a finite number of $M_{x}$ partial differential equations being coupled through the nonlinear convective and source term. These equations are then discretized using the finite volume method with first-order upwind scheme [4]. The semi-discrete form of the PBE describing droplet hydrodynamics is given by:

$$
\begin{aligned}
& \frac{d \phi_{i, l}}{d t}+\frac{F_{i, l+1 / 2}-F_{i, l-1 / 2}}{\Delta z_{l}}= \\
& \frac{\left.\left.D_{d}\left(\partial \phi_{i} / \partial z\right)\right|_{l+1 / 2}-D_{d}\left(\partial \phi_{i} / \partial z\right)\right]_{l-1 / 2}}{\Delta z_{l}}+\frac{Q_{d}}{A_{c}} \frac{\phi_{i}^{\text {feed }}}{\bar{V}_{d}} \frac{\delta_{l, i_{d}}}{\Delta z_{l}}+\rho\left(\phi_{l}, d\right)
\end{aligned}
$$

with $i=1,2, \ldots M_{x}$, and $l=1,2, \ldots L$. In the above equation the column height is discretized into a finite number of spatial cells whose width is $\Delta z_{l}$, which results in a system of ODEs of size: $M_{x} \times L$.

\section{HYDRODYNAMICS}

\subsection{Droplet Velocity}

The hydrodynamic behavior of droplets through their movement in a surrounding continuous phase is shown in Fig. (1). Depending on the droplet diameter and droplet velocity four different velocity boundary of the droplets in the surrounding continuous phase can be distinguished. The first boundary of droplet diameters identifies small droplets with rigid phase boundaries, which have no internal circulation. These droplets are moving as rigid spheres. For larger diameter there are shear forces at the droplet surface, so that an internal circulation begins to occur. Due to the circulation of the droplets they move faster than rigid spheres. For even larger diameter the droplets lose their spherical shape at the same time the droplet begin to oscillate and with further increase of the droplet diameter the droplet deforms and finally breaks up. The velocity of the droplets decreases as a result of the increasing flow resistance [35-37]. The droplet velocity and the axial dispersion are the key parameters for calculating the drift term in the population balance. The average rising velocity $V_{d}(z, d)$ of a droplet of diameter $d$, expressed as (see also Godfrey and Slater, [26]):

$V_{d}(z, d)=V_{r}(z, d)-V_{c}(z)$

where, $V_{r}(z, d)$ describes the relative velocity of droplets with diameter $d$ in a swarm influence, it is calculated by:

$V_{r}(z, d)=(1-\varphi(z))^{m} V_{r}^{*}(d)$

where $m$ is a variable exponent, which reflects the local hydrodynamic conditions and is called swarm exponent.

The swarm exponent $m$ takes slipstream effects into account. The swarm exponent as a function of the droplet Reynolds number; $V_{r}^{*}(d)$ refers to the relative velocity of a sin-

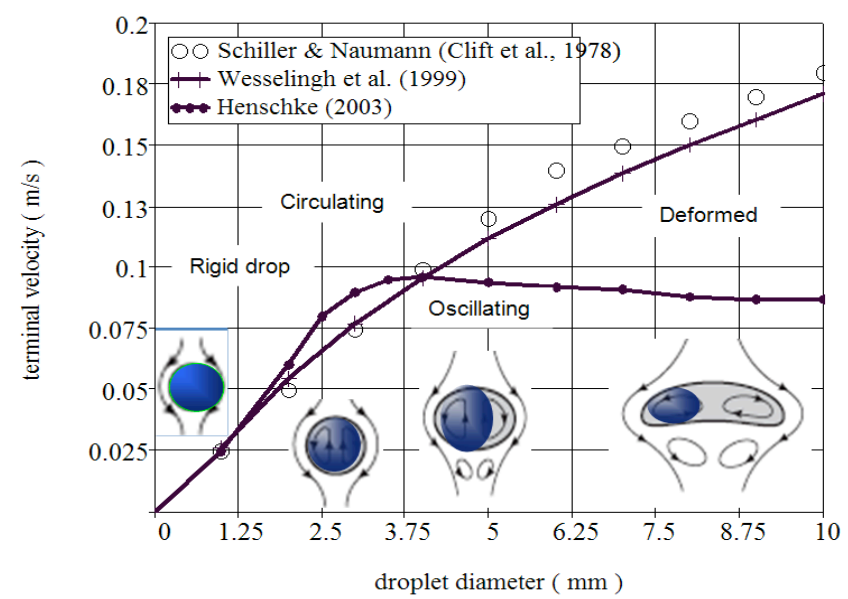

Fig. (1). Terminal velocity as function of droplets diameter. 
gle droplet in the column. The characteristic or free droplet rise velocity in an infinite medium is $V_{t}(d)$. The relative velocity of the single droplet is the product of free droplet rising velocity and slowing factor $K_{v}$ :

$V_{r}^{*}(d)=K_{v} V_{t}(d)$

The slowing factor takes into account the internal geometry of the column. A number of authors describe the suitability of velocity correlations for specific droplet size ranges or certain physical properties [26,37-40]. A selection of these velocity correlations has been implemented in simulation program.

\subsubsection{Velocity Models}

The LLECMOD simulation program allows the user to conduct the simulation with different approaches to calculate the free rise velocity of the droplets. The slowing factor $K_{v}$ depends on the selected column type and it is calculated internally in the program. The relative velocity is calculated for each droplet size in the program block user function of the active module and evaluated in the program solver part. The required data for free rising velocity calculation are available in the solver and will be passed when calling the SDVelm subroutine. In the Solver the droplet swarm velocity is calculated using the relative velocity of the droplets.

The model proposed by Henschke [37] for droplet velocity is calculated by combining the velocities of spherical, deformed, and oscillating droplets. The proposed model gives a continuous function over the whole diameter range. For spherical droplets or rigid bubbles the droplet velocity is given by:

$$
v_{\text {spherical }}=\frac{R e_{\infty, \text { spherical }} \mu_{c}}{\rho_{c} d}
$$

The velocity of very large droplets or bubbles is investigated experimentally and describes both droplets and bubbles and given by:

$$
v_{g r}=\sqrt{\frac{\Delta \rho g d}{2 \rho_{c}}}
$$

with oscillating droplets the velocity is calculated by:

$v_{o s}=\sqrt{\frac{2 a_{15} \gamma}{\rho_{c} d}}$

where the parameter $a_{15}$ is adjusted to measured values. The transition function describes the velocity over the transition region of oscillating to deformed drops:

$$
v_{o s, g r}=\left(v_{o s}^{\alpha_{g r}}+v_{g s}^{\alpha_{g r}}\right)^{1 / \alpha_{g r}}
$$

The author suggesting exponent $\alpha_{g r}=8$. The droplet velocity for all states droplet can be specified by the following equation:

$$
V_{t}=\frac{v_{o s, g r} v_{\text {rund }}}{\left(v_{o s, g r}^{a_{15}}+v_{\text {rund }}^{a_{16}}\right)^{1 / a_{16}}}
$$

The exponent $\alpha_{15}$ describes the sharpness of the transition between sub-models $\alpha_{g r}$ of Eq. (13). Fig. (2) presents the input parameters for Henschke [37] velocity model and three parameters $a_{16}, a_{15}$ and $d_{u m}$ have to be adapted to measure values. Fig. (3) shows the input window for the constants of

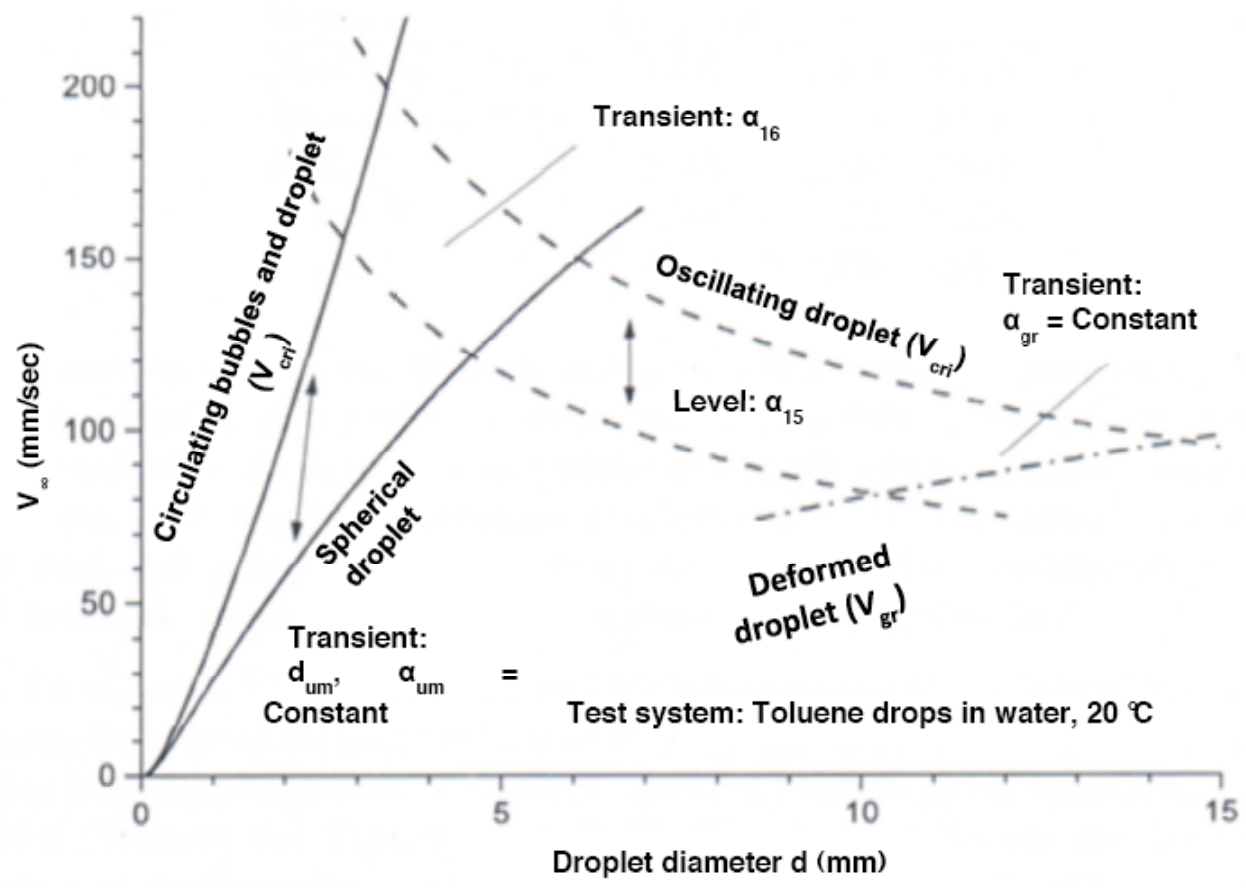

Fig. (2). Parameters for the modeling of single droplet velocity [37]. 
the Henschke [37] velocity model. The depicted coefficients are equivalent to those used in the calculation of the test system butyl acetate-water- acetone in a pulsed packed extraction column.

\subsubsection{Influence of Internals (Packing and Sieve Plate)}

In a column with internals the droplets rise slowly. The deceleration effects come from collisions with internals and from an extension of the droplet path. In practice, this effect is expressed by a slowing factor $k_{v}$; the ratio of droplet velocities with internals $v_{\text {char }}$ and without internals $v_{\infty}$. By definition, the value of slowing factor is between 0 and 1 .

$k_{v}=\frac{v_{c h a r}}{v_{\infty}}$

In the literature, there are several correlations for different geometries. In LLECMOD those equations have been implemented, which were validated for a wide range of parameters. For the description of the droplet motion in pulsed packed a correlations developed by Garthe [41] is applied.

$k_{v}=0.077 \pi_{H_{P} K}^{0.138} \pi_{a_{P} K}^{-0.566} \pi_{d}^{-0.769} \pi_{\sigma}^{0.184}\left(1+\pi_{a f}\right)^{0.08}$

Based on the analysis of experimental results Garthe [41] developed a new correlation for pulsed sieve plate column:

$k_{v}=1.406 \varphi_{s t}^{0.145} \pi_{\sigma}^{-0.028} \exp \left(-0.129\left(\frac{\mathrm{d}}{\mathrm{d}_{\mathrm{h}}}\right)^{1.134}\left(1-\varphi_{s t}\right)^{-2.161}\right)$

The dimensionless numbers $\pi_{i}$ are calculated as follows [41]: The dimensionless height of a packing: $\pi_{h_{P_{K}}}=h_{p_{k}}\left(\frac{\rho_{c} \Delta \rho g}{\mu_{c}^{2}}\right)^{1 / 3}$, the dimensionless volumetric surface area of a packing: $\pi_{a_{P_{K}}}=a_{P_{k}}\left(\frac{\mu_{c}^{2}}{\rho_{c} \Delta \rho g}\right)^{1 / 3}$, the dimen-

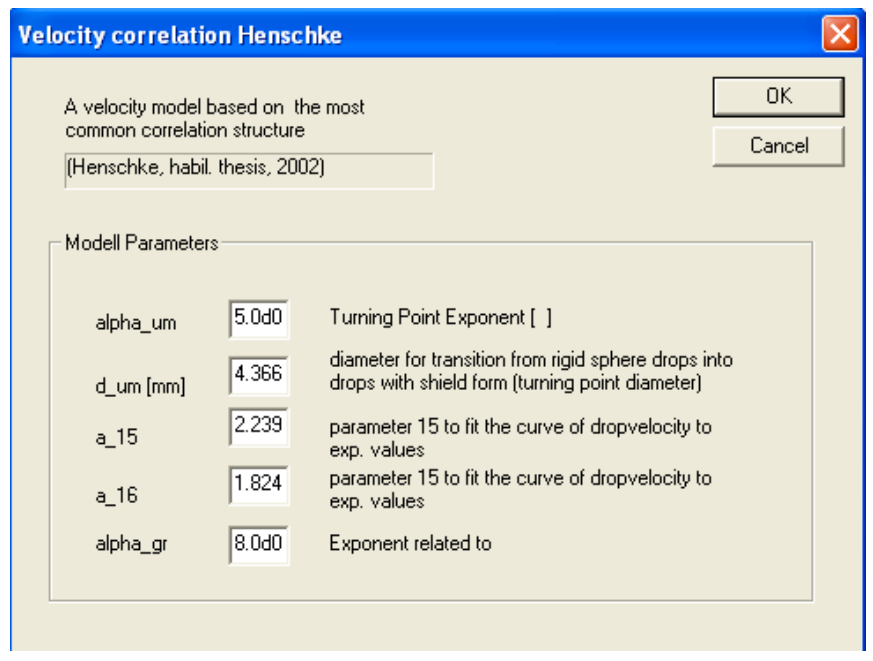

Fig. (3). Input parameters for Henschke [37] velocity model. sionless droplet diameter: $\pi_{d=} d\left(\frac{\rho_{c} \Delta \rho g}{\mu_{c}^{2}}\right)^{1 / 3}$, the dimension-

less interfacial tension: $\pi_{\sigma}=\sigma\left(\frac{\rho_{c}^{2}}{\mu_{c}^{4} \Delta \rho g}\right)^{1 / 3}$ and the dimen-

sionless pulsation intensity: $\pi_{a f}=a f\left(\frac{\rho_{c}^{2}}{\mu_{c} \Delta \rho g}\right)^{1 / 3}$.

\subsubsection{Swarm Effect}

Through surrounding droplets the velocity of individual droplet is slowed. In LLECMOD different models are used for different types of internals. To calculate the swarm velocity in pulsed columns often purely empirical equations are used. The equation of Gaylor et al., [42] is applied, which assumed $n$ to be a unity:

$v_{r s}=v_{\text {char }}(1-\varepsilon)^{n}$

For packed columns Garthe [41] suggested an equation depending on droplet diameter and free rise velocity:

$n=16.2\left(\frac{v_{\text {char }} \rho_{c} d}{\mu_{c}}\right)^{-0.54}-1$

\subsection{Droplet Breakage}

\subsubsection{Droplet Breakage in Pulsed Sieve Plate Column}

To investigate the droplet breakage Haverland [43] has used an experimental setup, shown in Fig. (4). The generated droplets first rising through a tube, when the rising droplets reach the sieve tray it is possibly to break up into multiple daughter drops. These daughter droplets rise to the large collecting funnel and an automatic droplet size measurements is supplied. The breakage behavior of a test system in a certain sieve tray can be characterized as shown in Fig. (5). Droplets with diameters below the stable droplet diameter $d_{\text {stab }}$ do not split. The concept of stable droplet diameter describes a droplet that will not split even at arbitrarily long residence time in the column [43]. Large droplets with diameters above the $d_{100}$-line are split to $100 \%$. Between the $d_{s t a b}$-line and the $d_{100}$-line the breakage probability $p_{S}$ depends on the test system of physical prosperities, the droplet diameter $d$, the stable boundary diameter $d_{s t a b}$, the terminal velocity $V_{a b s}$ and the pulsation intensity af which increased from $0 \%$ to $100 \%$. There are different effects depending on droplet diameter and pulsation that may lead to droplet breakage. Even without any internals and pulsation breakage occurs when a certain size is exceeded and the small droplets pass through the sieve holes without breakage.

Fig. (5) illustrates the behavior of droplets in a sieve plate with a hole diameter of $2 \mathrm{~mm}$. The lines $d_{1}$ and $d_{2}$ are generated by the variation of the pulsation intensities. With increasing the pulsation the droplets reach the area where droplet breakup occurs with diameter $d_{s t a b}=d_{L}$. These droplet deformations occur noticeably slow inside the sieve plate 


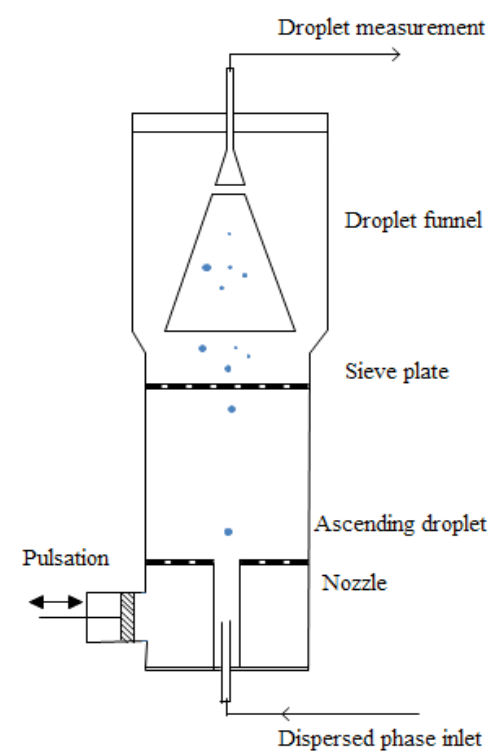

Fig. (4). Experimental setup to investigate the breakage of single droplets [43].

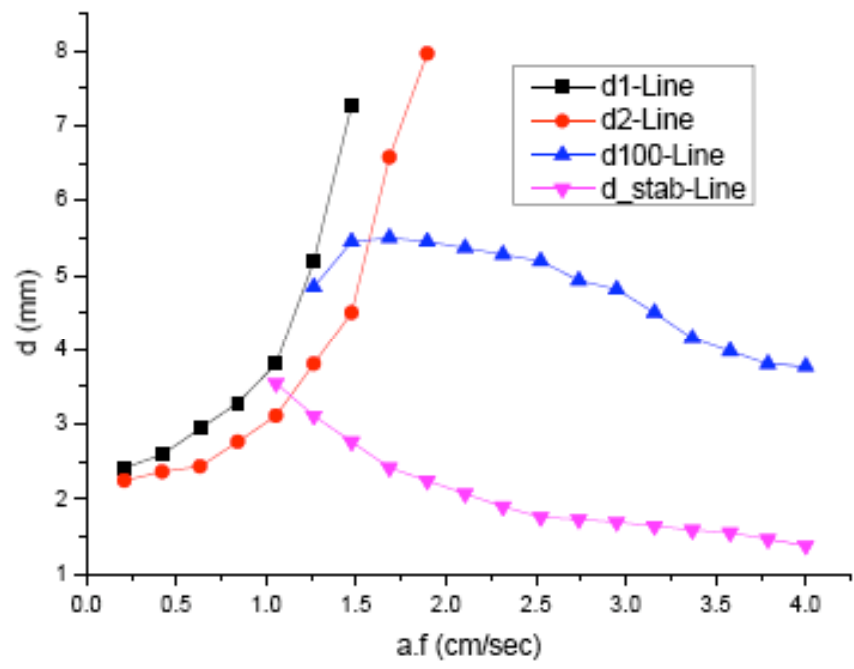

Fig. (5). Characterization of the single droplet breakage in sieve trays [43].

holes. With further increasing pulsation $d_{\text {stab }}<d_{L}$ is achieved. According to Haverland [43] an area exists by in which the droplets are broken at the bridge between two holes. At very high pulsation intensities finally a point is reached at which the droplets will not probably be directed right on the sieve plate but split in the turbulence field near a perforated bottom.

\subsubsection{Mean Number of Daughter Droplets Per Breakage}

For the measurements of Haverland [43] the mean number of daughter droplets $n_{Z}$ was inter alia determined for each breakup. Here just the breakup of mother droplets was evaluated. These measurements thus give no statement about the breakage probability $p_{S}$ or the actual number of daughter droplets $n_{Z}$. The work of Haverland [43] showed that only the mother droplets larger than the stable droplet diameter $d_{s t a b}$, are divided into two or more daughter droplets. With increasing the mother droplets size and by increasing pulsation intensity the number of daughter droplets is increasing $\left(\bar{n}_{z} \leq 20\right)$. A simple empirical approach to calculate the mean number of daughter droplets is given by Henschke, [37]:

$\bar{n}_{z}=2.0+\xi_{3}\left(\left(\frac{d^{\prime}}{d_{\text {stab }}}\right)^{\xi_{4}}-1\right)\left(\frac{a f}{\Phi v_{\infty}}+1\right)^{\xi_{5}}$

The adjustment parameter $\xi_{i}$ resulted in the following: $\xi_{3}=0.3, \xi_{4}=2.0$ and $\xi_{5}=2.0$

\subsubsection{Daughter Droplet Distribution}

The daughter droplet distribution used to determine the number of daughter droplets which results from the breakage function of many mother drops. For pulsed sieve plate extraction column, Henschke's [37] approach is used to evaluate droplet size distribution. In this approach all parameters that influence the stable droplet diameter are considered. The breakage probability is the number of daughter droplets per breakage and daughter droplet distributions [37].

$$
\begin{aligned}
& S=3 \bar{n}_{z}\left(\bar{n}_{z}-1\right)\left(1-\left(\frac{d}{d^{\prime}}\right)^{3}\right)^{\left(\bar{n}_{z}-2\right)}\left(\frac{d}{d^{\prime}}\right)^{\left(5 / d^{\prime}\right)}\left(\frac{d}{d^{\prime}}\right)^{3} \\
& n_{z}=\left(\bar{n}_{z}-\left(\frac{0.2}{\bar{n}_{z}-1.8}\right)\right)(1+(1-\sqrt{S}))
\end{aligned}
$$

Here, $d_{\text {stab }}$ is the stable droplet diameter, where below this diameter no more breakage occurred, which is calculated according to Henschke [37] as:

$d_{s t a b}=\frac{d_{s t a b, 1234} d_{s t a b, 5}}{\left(d_{s t a b, 1234}^{20}+d_{s t a b, 5}^{20}\right)^{1 / 20}}$

where $d_{s t a b, 1234}$ is the stable droplet diameter as a result of combination of four different models of stable droplet diameter to take into considerations all the effects that might affect the droplet diameter, and $d_{s t a b, 5}$ is the fifth model. These models are described in detail in Henschke [37].

\subsubsection{Henschke (2004) Breakage Probability in Pulsed Sieve Plate Column}

By knowing the mean number of daughter droplets per breakup event, it is not clear whether there is any the mother droplet breakup at all. It is known so far that only droplet with the diameter limit $d_{\text {stab }}$ has a breakage probability tending to zero, and that an area exists above a $d_{100}$-line in which all the droplets are breakup. With these boundary conditions and the assumption that the breakage probability correlates with the mean number of daughter droplets $\bar{n}_{z}$, the breakage probability is given by Henschke [37]:

$$
P s=\xi_{6}\left(1-\exp \left[\xi_{7}\left(\bar{n}_{z}-2\right)\right]\right), \quad \xi_{6}=1.05 \text { and } \xi_{7}=-0.45
$$


In the measurement of Haverland [43] the parameters $\xi_{6}$ and $\xi_{7}$ are adjustable parameters for the breakage probability $P s$, in this case $n_{Z}$ is taking into calculation with the stable boundary diameter which is calculated using Henschke [37] model. Fig. (6) shows the breakage probabilities for different mother droplet diameter and pulsation intensities in pulsed sieve plate column. It is seen that the breakage probability of small mother droplet $\left(d_{m}=2 \mathrm{~mm}\right)$ is relatively rising slowly with the pulsation intensity, while with the big mother droplets $\left(d_{m}=5 \mathrm{~mm}\right)$ one is experiencing almost a jump-rise. This trend is also reproduced by the model correctly. However, clear deviations occur at the largest mother droplet diameter.

Fig. (7) depicts the input window for the constants of the Henschke [37] breakage model for pulsed sieve plate column. The depicted coefficients are equivalent to those used in the calculation of the test system water-acetone-butyl acetate (w-a-b) in a sieve plate extraction column.

\subsubsection{Garthe [41] Breakage Probability in Pulsed Sieve Plate Columns}

The breakage of single droplets in pulsed sieve plate extraction columns depends on the geometry of the internals and the energy input. In pulsed sieve tray columns droplet breakage is basically governed by the size of the sieve tray holes and the pulsation intensity, see Haverland [43] and Wagner and Blaß [44]. The breakage probability in pulsed sieve tray compartments can be predicted by a simple correlation introduced by Haverland [43]:

$$
P_{B}\left(d_{m}\right)={\frac{\left(d_{m}-d_{s t a b}\right)}{\left(d_{100}-d_{s t a b}\right)}}^{C}
$$

Where $d_{100} \& d_{s t a b}$ are the characteristic droplet diameters and $C$ is a constant depending on the pulsation intensity. The disadvantage of this correlation is that the exponent $C$ has to be determined for each individual pulsation intensity and that extrapolation to other pulsation intensities is difficult. To overcome this difficulty Garthe [41] has developed a new correlation based on the above equation as:

$P_{B}\left(d_{M}\right)=C_{1} \pi_{a f}^{C_{2}} \frac{\left(d_{m}-d_{s t a b}\right) /\left(d_{100}-d_{s t a b}\right)^{C_{3}}}{C_{4}+\left(d_{m}-d_{s t a b}\right) /\left(d_{100}-d_{s t a b}\right)^{C_{3}}}$

where $\pi_{a f}$ is a dimensionless number taking into account the influence of the pulsation intensity on the breakage probability and is given by:

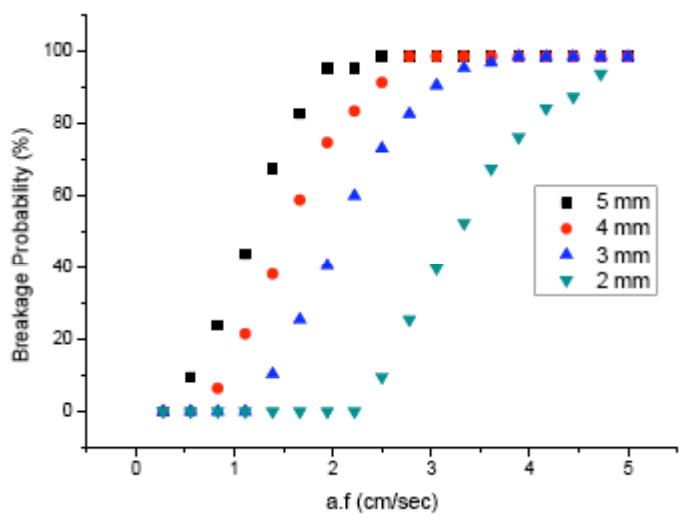

Fig. (6). Droplets breakage probability as a function of pulsation intensity.

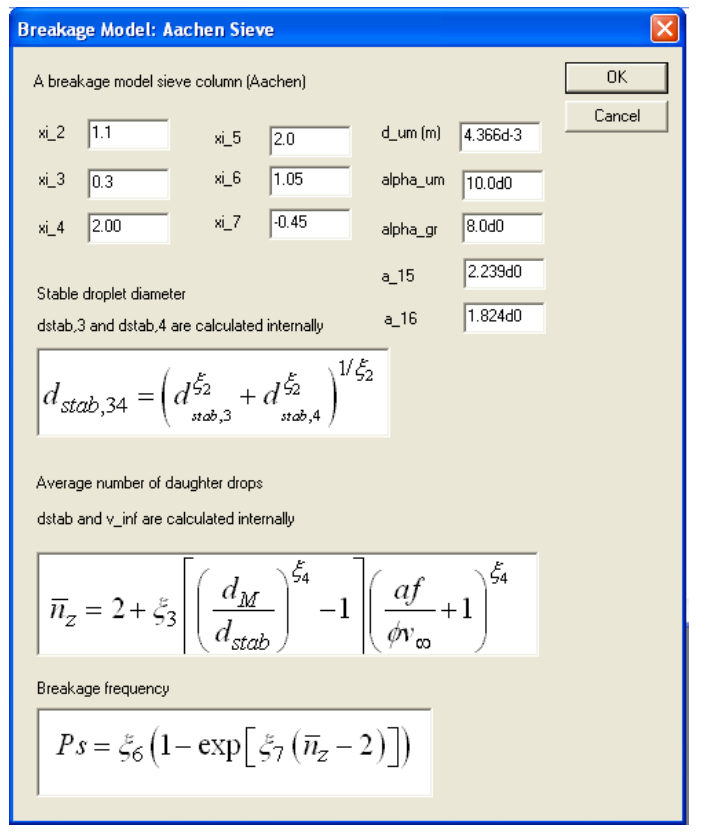

Fig. (7). Input dialog for breakage Henschke [37] model in pulsed sieve plate columns.

$\pi_{a f}=a . f \cdot\left(\frac{\rho_{c}^{2}}{\mu_{c} \cdot \Delta \rho . g}\right)^{1 / 3}$

The new correlation describes the breakage in a sieve tray compartment with only one set of constant factors for a liquid-liquid system. The constant $C_{i}$ factors in Eq.(26) are listed in Table 1. To predict the breakage probability in sieve tray compartments with Eq. (26), the characteristic droplet

Table 1. Garthe [41] Breakage Probability Constants in Pulsed Sieve Plate Columns

\begin{tabular}{|l|l|l|l|l|l|}
\hline \multicolumn{1}{|c|}{ Sieve Tray } & \multicolumn{1}{|c|}{ Test System } & \multicolumn{1}{c|}{$\boldsymbol{C}_{\boldsymbol{1}}$} & \multicolumn{1}{c|}{$\boldsymbol{C}_{2}$} & \multicolumn{1}{c|}{$\boldsymbol{C}_{3}$} & \multicolumn{1}{c|}{$\boldsymbol{C}_{4}$} \\
\hline \hline \multirow{2}{*}{$2 \mathrm{~mm}$} & water-acetone-toluene (d) & 3.81 & 0.61 & 1.11 & 3.47 \\
\cline { 2 - 7 } & water-acetone-butyl acetate (d) & 2.49 & 0.27 & 0.95 & 1.77 \\
\hline $3.2 \mathrm{~mm}$ & water-MIBK (d) & 2.49 & 0.27 & 0.95 \\
\hline
\end{tabular}


diameters $d_{100} \& d_{s t a b}$ have to be experimentally determined for each pulsation intensity. According to the definition, $d_{s t a b}$ is determined by a droplet with a breakage probability somewhere between $0<P_{B} \leq 0.03$ and the diameter $d_{100}$ is given by a droplet with a breakage probability somewhere between $0.97<P_{B} \leq 1.00$. The data for the characteristic droplet diameters $d_{100} \& d_{s t a b}$ for sieve trays are given in Garthe [41].

\subsubsection{Droplet Breakage in Pulsed Packed Column}

\subsubsection{Daughter Droplet Size Distribution}

The daughter droplet size distribution used to determine the number of daughter droplets which results from the breakage function of many mother drops. For pulsed packed extraction column the daughter droplet distribution is assumed to follow the beta distribution function $\beta\left(d, d^{\prime}\right)$, which is given by Bahmanyar and Slater [45], where $v$ is the average number of daughter droplets per breakage:

$\nu=2+0.34\left(\left(\frac{d^{\prime}}{d_{s t a b}}\right)-1\right)^{1.96}$

$\beta\left(d / d^{\prime}\right)=3 \nu(\nu-1)\left(1-\left(\frac{d}{d^{\prime}}\right)^{3}\right)^{\nu-2}\left(\frac{d^{5}}{d^{\prime 6}}\right)$

3.2.2.2. Henschke Breakage Probability in Pulsed Packed Column

The model of droplet breakage in structure packing columns based on the following idea: a droplet in the flow through the column from the bottom up to the edges or intersection points of the packing elements and its upward movement is stopped. Since the droplet usually is not exactly centred on the obstacle to form two different-sized parts, which at the moment are still connected via a liquid film. Since the two parts of the original droplet are usually on different sides of the packing sheet, they will continue their way through the column independently of each other and can be considered as new droplets after being split. In oblique serrated sheets of packages there are three places as a dispersing points: the lower plate, the packing crossing points and the hole edges in the case of cross-drilled, well-ordered packing. When the droplet enters a packing element it might coalesce with the plate bottom or breakup by it. The entire length of the edge as a possible place of breakage comes into question. It is not a discrete point in the cross section packing. Each packing intersection is a potential place of breakage. For a given test system, the droplet breakage depends on the geometry of the structure packing, pulsation intensity and the size of the mother droplet. In extraction, different types of packings can be used from various manufacturers. The packing type is characterized by geometric parameters the packing structure, the package volume-surface, the angle of the packing sheets or the pack channels, element height, the holes and other manufacturers' specific dimensions.
In the pulsed packed column, the maximum droplet velocity $v_{\max }$ consists of two parts. The first relies on the characteristic velocity of the droplet relative to a stationary reference point in the column flow effects such as wall effects, swarm behavior or the packing themselves affect the relative motion. The second is caused by the maximum pulsation, thus it is:

$v_{\max }=\pi a f+v_{c h r}$

Under the condition of mass conservation and constant density with the below parameters $n_{T T}$, the relationship between daughter droplet size $d_{T T}$ and mother droplet $d_{m}$ is described as follows:

$d_{T T}=\left(\frac{1}{n_{T T}}\right)^{1 / 3} d_{m}$

For a description of the parameter $n_{T T}$ the following approach was chosen:

$n_{T T}=1.06388+2.062556 \sqrt{\frac{\Delta \rho g d_{m}^{2}}{\sigma}}$

With these relations an equation for the mother droplet diameter $d_{m}$ which is being split yet is obtained, and thus corresponds $d_{\text {stab }}$, and $d_{\text {stab }}=d_{\mathrm{m}}$. This equation can be solved iteratively [37].

$d_{s t a b}^{2}+d_{s t a b} \frac{\left(\pi a f+v_{a b s}\right)^{2}\left(\rho_{c}+0.6 \rho_{d}\right)}{2\left(\rho_{c}-\rho_{d}\right) g}-\frac{6 \sigma\left(n_{T T}^{1 / 3}-1\right)}{\left(\rho_{c}-\rho_{d}\right) g}=0$

If the diameter of a droplet in the column is smaller than the stable droplet diameter $d_{\text {stab }}$, then the breakage probability according to the definition given above is equal to zero. But if the diameter is larger than the stable droplet diameter then the all influencing factors which determine the droplet breakage are summarized in the so-called local breakage probability $P_{S}$, that characterizes the droplet $i$ in the current time interval.

The dependence of the breakage probability $P_{S}$ on the pulsation intensity and the droplet velocity can be specified only qualitatively. Basically, it is expected that at higher pulsation intensity a higher breakage probability occurs. This influence cannot be explained precisely and a linear increase of the breakage probability with the pulsation intensity and the relative velocity is assumed [46]:

$P_{S} \sim\left(2 a f+v_{a b s}\right)$

The current droplets velocity $v_{a b s}$ is recognized as depending on holdup and location of the droplet to pack against a stationary reference point. The influence of pulsation is determined by the average pulsation speed $2 a f$, also the amount in the pulsation average velocity is recorded over a period. The relationship between the breakage probability $P_{S}$ and the mother droplet diameter $d_{M}$ in the light of the stable droplet diameter $d_{\text {stab }}$ is taken into consideration; the following correlation of Leu [46] can be used as the basis for the breakage probability: 


$$
P_{S} \sim\left(\frac{d_{\mathrm{m}}-d_{\mathrm{stab}}}{d_{\mathrm{m}}}\right)^{a_{1}}
$$

The exponent $a_{1}$ is considered as a principle parameter. In the experimental adaptation a value of $a_{l}=1.5$ has proved to be useful. The proportionality factor $k_{S}$ is introduced to capture the influences of the chemical system used. Henschke [37] summarized all the parameters which might have influenced the local breakage probability $P_{S}$ and developed the following equation:

$P_{S}=k_{S} N\left(2 a f+v_{a b s}\right) d_{m}\left(\frac{d_{\mathrm{m}}-d_{\mathrm{stab}}}{d_{\mathrm{m}}}\right)^{a_{1}}$

The proportionality factor $k_{S}$ and the exponent $a_{l}$ can by fitted to the experimental data. The local breakage probability $P_{S}$ is given below for various locations within the column.

Slats edge: The local breakage probability; $P_{S, L U K}$ at the lower slats edge [37]:

$P_{S, L U K}=K_{S} \frac{\pi}{8} d_{m} a_{P K}\left(2 a f+v_{a b s}\right) d_{m}\left(\frac{d_{\mathrm{m}}-d_{\mathrm{stab}}}{d_{\mathrm{m}}}\right)^{a_{1}}$

Lamellae crossing point: The local breakage probability $P_{S, L K P}$ on the lamellae crossing points [37]:

$P_{s}=k_{s} \frac{\pi}{8} \frac{\Delta h_{T} d_{m}^{2} \sin (2 \alpha)}{x^{2} z}\left(2 a f+v_{a b s}\right) d_{m}\left(\frac{d_{m}-d_{s t a b}}{d_{m}}\right)^{a_{1}}$

Fig. (8) depicts the input window for the constants of the Henschke [37] breakage model for pulsed packed column. The depicted coefficients are equivalent to those used in the calculation of the test system water-acetone-butyl acetate (w$\mathrm{a}-\mathrm{b})$ in a packed extraction column.

\subsubsection{Garthe Breakage Probability in Pulsed Packed Columns}

Leu 1995 has investigated the breakage of single droplets in a pulsed packed column. Packing with a high volumetric surface area possess more breakage opportunities and result in a higher breakage probability. Increasing the mother droplet diameter and the pulsation intensity causes an increase of the breakage probability. Also, the interfacial tension has large influence on the droplet breakage. For the same operating conditions the large difference in the breakage probabilities is explained by the significantly lower interfacial tension. Generally, the lower is the interfacial tension; the higher is the breakage probability. Garthe [41] showed that, due to the complex mechanisms that influence the breakage of single droplets in pulsed compartments, no general model could be developed to predict the characteristic droplet diameters $d_{100} \& d_{s t a b}$ together with the breakage probability. However, the new correlation (see Eq. (26)) allows the determination of the breakage probability just from knowledge of the characteristic diameters. Therefore, the new correlation leads to a significant reduction of the experimental effort which is in the same form as for sieve tray column. To predict the breakage probability in Montz packing compartments with Eq. (26), the characteristic droplet diameters

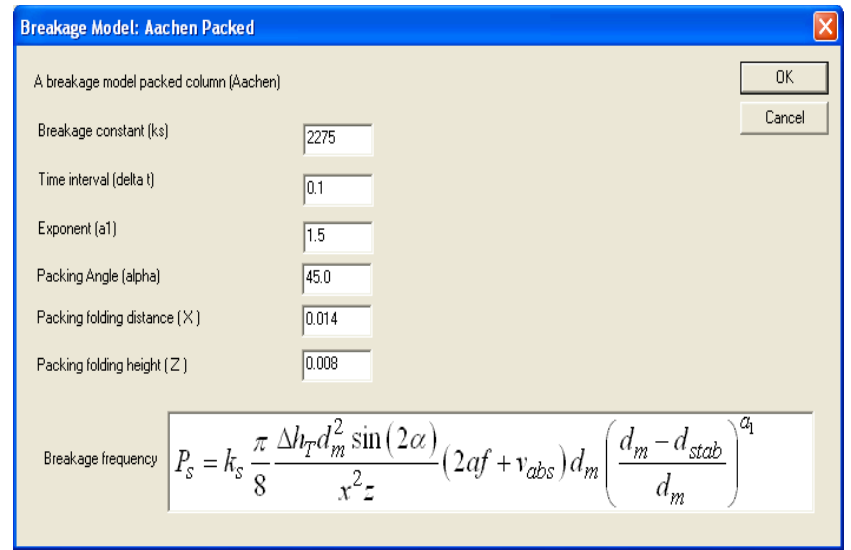

Fig. (8). Input dialog for Henschke [37] breakage model in pulsed packed column.

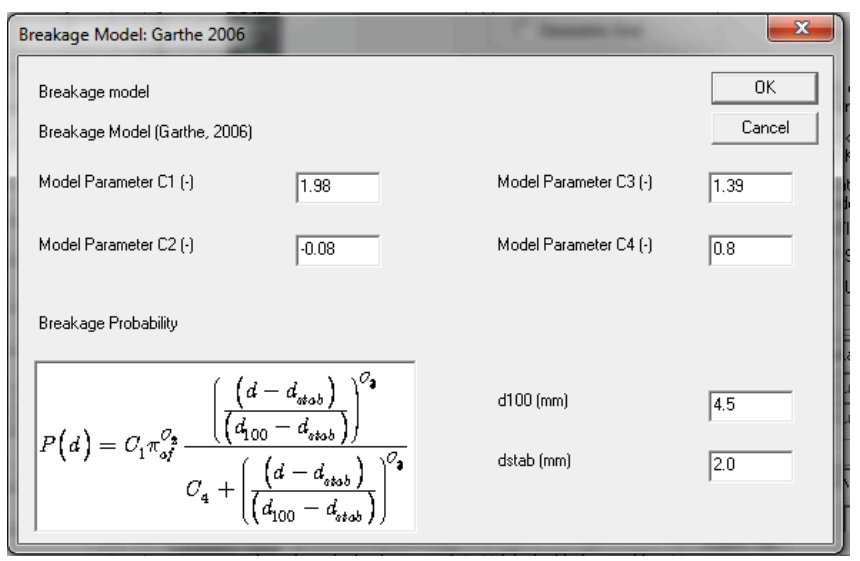

Fig. (9). Input dialog for Garthe [41] breakage model in pulsed (packed and sieve plate) columns.

$d_{100} \& d_{s t a b}$ have to be determined experimentally for each pulsation intensity. According to the definition, $d_{s t a b}$ is determined by a droplet with a breakage probability somewhere between $0<P_{B} \leq 0.03$ and the diameter $d_{100}$ is given by a droplet with a breakage probability somewhere between $0.97<P_{B} \leq 1.00$. The data for the characteristic droplet diameters for Montz packing investigated are listed elsewhere [41], the probability constants are summarized in Table 2.

Fig. (9) depicts the input window for the constants of the Garthe [41] breakage model for pulsed (packed and sieve plate columns). The depicted coefficients are equivalent to those used in the calculation of the test system wateracetone-toluene (w-a-t) in a pulsed packed extraction column.

\subsection{Droplet Coalescence}

Coalescence is the process by which two or more droplets or particles merged during contact to form a single daughter droplet (bubble) either (droplet-dropletcoalescence) or a droplet with its main phase (dropletinterfacial coalescence). For the droplet-droplet-coalescence as well as for the droplet-interfacial-coalescence the droplet 
coalescence process can be approximated in three steps: film drainage, film rupture and break down and then coalescence.

\subsubsection{Coalescence in Pulsed Sieve Plates Columns}

The modeling of coalescence is based on observations for droplet growth within a densely packed layer. There, the droplets grow by droplet-droplet coalescence of a Sauter diameter $d_{32,0}$ to a top diameter of $d_{32, e}$. The temporal variation of the Sauter diameter at any point within the densely packed layer may be specified with the droplet average life $\tau_{K}$ by the correlation of Hartland and Vohra [47]:

$$
\frac{\partial d_{32}}{\partial t}=\frac{d_{32}}{6 \tau_{k}}
$$

This is affected by the liquid-liquid system characteristic physical properties and the Sauter diameter but is independent of individual droplet sizes. For the determination of the mean droplet diameter different correlations are given in the literature. The study of Smith and Davies [48] and Hozawa et al., [49] indicate that the droplet lifetime behaves approximately inversely proportional to the Sauter diameter. Henschke [50] shows that $\tau_{K} \sim d_{32}{ }^{1 / 3}$. Therefore, the below correlation is for the determination of the mean droplets life time with Hamaker coefficient $H_{c d}$ [37]:

$$
\tau_{k}=2.05 \frac{\mu_{c} d^{1 / 3}}{\sigma^{1 / 3} H_{c d}^{1 / 6}(\Delta \rho g)^{1 / 2} r_{K}^{+}}
$$

The asymmetry parameter $\mathrm{r}_{\mathrm{K}}^{+}$is dependent on the test system used. In this model, there is no dependence on the holdup or the droplet diameter taken into account. The asymmetry parameter $\mathrm{r}_{\mathrm{K}}{ }^{+}$integrated within the constant $\xi_{8}$. Henschke [37] takes into account the coalescence time behavior which is inversely proportional to the holdup. Therefore the model is expanded to:

$$
\tau_{k}=\xi_{8} \frac{\mu_{c} d^{1 / 3}}{\varepsilon \sigma^{1 / 3} H_{c d}^{1 / 6}(\Delta \rho g)^{1 / 2}}
$$

The $\xi_{8}$ parameter is adapted to laboratory experiments. For the system toluene (d) and water (c) and a mass transfer from continuous phase to the dispersed phase then $\xi_{8}=2500$ according to [37]. Coalescence rate analysis and modeling has been paid greater attention. Many authors proposed phenomenological models based on the concept of collision frequency and coalescence efficiency, where the latter assumed to be a function of the two coalescing droplets. Thus, the coalescence model after Henschke [37] is modified to take into account the size of the two coalesce droplets [10]. In this work, the modified coalescence model is used in the simulation of both pulsed (packed and sieve plate) columns:

$$
\omega\left(d, d^{\prime}\right)=c\left(v(d)^{n}+v\left(d^{\prime}\right)^{n}\right) \frac{\phi_{y} \sigma^{1 / 3} H_{c d}^{1 / 6}(\Delta \rho g)^{1 / 2}}{\xi_{8} \mu_{c} d^{1 / 3}}
$$

In this modified coalescence frequency $\omega\left(d, d^{\prime}\right), d, d^{\prime}$ is the droplet diameter, $v$ is the droplet volume, $\phi_{y}$ is the dispersed phase holdup, $\sigma$ is the mixture interfacial tension and $H_{c d}$ is the Hamaker constant, the value used in the simulation is $10 \mathrm{e}-20$. The adjustable parameter $\xi_{8}$ was fitted to experimental data for the two standard EFCE test systems: water-acetone-toluene and water-acetone-butyl acetate and is given by Henschke [37]. The values for this parameter are 2500 and 1500 for the first and second system respectively. Adjusted constant $c$ values used in fitting column hydrodynamics to the experimental data [41] are listed in Table 6.

\subsubsection{Coalescence in Pulsed Columns with Structure Packing}

The determination of the coalescence in structured packings can be considered likewise as in the sieve plates. For the mass transfer direction from the continuous phase to the dispersed phase the coalescence plays only a minor role in the overall process being confirmed by Rohlfing [51]. Therefore, the coalescence is determined by the same equations as those described in Section 3.3.2. The constant $\xi_{8}$ in this turn is adapted to experimental data [52].

\subsubsection{Coalescence in Pulsed Columns with Random Packing}

More detailed coalescence investigations were carried out by Klinger [52]. The equations for the coalescence probability using the results of the laboratory cell measurements are described below. For the determination of the coalescence operations in a packed column it is assumed that the coalescence takes place only in the area of the packing. This is due to that the droplets lose in this area a part of their velocity because of the packing, coalesce can be obtained with the subsequent droplets. For the coalescence probability $P_{K}$ must continue to apply: $0 \leq P_{K} \leq 1$. Klinger [52] developed an empirical approach; therefore, the coalescence probability can be determined as a function of droplet diameter $d$, holdup and coalescence constant $C_{K}$, which reflects the influence of the physical properties:

$P_{K}=C_{K} d_{T r}^{k 1} \varepsilon^{k 2} \Delta t$

The increase of holdup on the coalescence increases the probability. The influence of the droplet diameter on the coa-

Table 2. Garthe [41] Breakage Probability Constants in Pulsed Packed Columns

\begin{tabular}{|c|l|l|l|l|l|}
\hline \multicolumn{1}{|c|}{ Packing } & \multicolumn{1}{|c|}{ Test System } & \multicolumn{1}{|c|}{$\mathbf{C}_{\mathbf{1}}$} & $\mathbf{C}_{\mathbf{2}}$ & \multicolumn{1}{|c|}{$\mathbf{C}_{\mathbf{3}}$} & \multicolumn{1}{c|}{$\mathbf{C}_{\mathbf{4}}$} \\
\hline \hline \multirow{3}{*}{ Montz-Pak B1-350 } & water-acetone-toluene (d) & 1.98 & -0.08 & 1.39 \\
\cline { 2 - 7 } & water-acetone-butyl acetate (d) & 1.06 & -0.07 & 0.8 \\
\cline { 2 - 7 } & Industrial test system & 1.06 & -0.07 & 2.99 & 0.13 \\
\hline
\end{tabular}


Table 3. Henschke [37] Coalescence Model Constants [52]

\begin{tabular}{|l|l|l|}
\hline \multicolumn{1}{|c|}{ Test System } & \multicolumn{1}{c|}{ Measuring Cell } & \multicolumn{1}{c|}{ C $_{\mathbf{K}}(\mathbf{s})$} \\
\hline \hline n-butanol (d) + water & Lab measuring cell & 2.25 \\
\hline n-butyl acetate (d) + water & Lab measuring cell & 1.00 \\
\hline toluene (d) + water & Lab measuring cell & 0.40 \\
\hline n-butyl acetate (d) + water & Pilot plant column & 1.00 \\
\hline
\end{tabular}

lescence is according to the following phenomena. The deformation of the boundary layer during the so-called film drainage varies for different droplet diameters. In addition, with increasing droplet diameter the rates of ascent and movement behavior are changing in the random packing. Table 3 shows the values for the coalescence constant $C_{K}$ for the test systems n-butyl acetate (d)- water (c), toluene (d)water (c) and n-butanol (d) -water (c). Fig. (10) depicts the input window for the constants of the modified Henschke [37] coalescence model for pulsed (packed and sieve plate) column. The depicted coefficients are equivalent to those used in the simulation of the test system toluene wateracetone-toluene (w-a-t) in a pulsed packed extraction column.

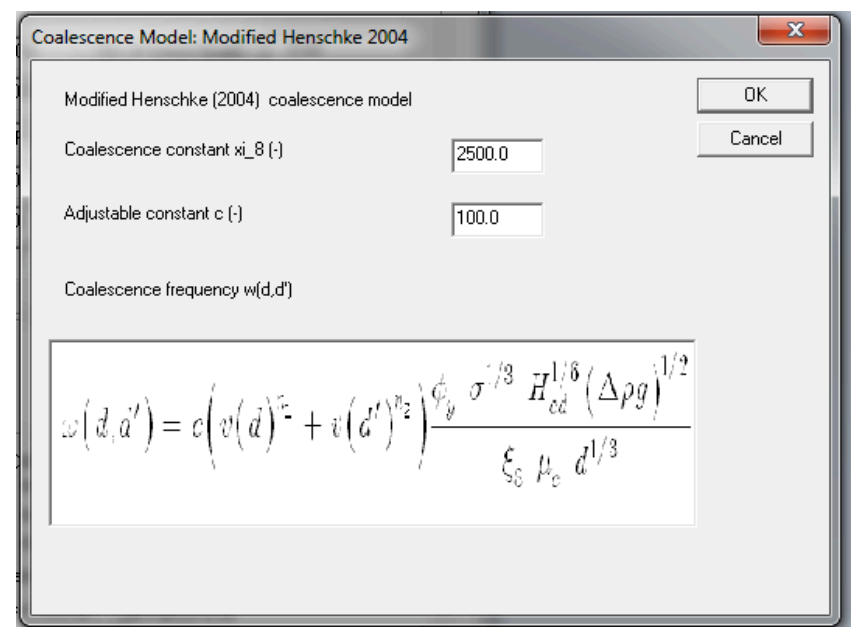

Fig. (10). Input dialog for Henschke [37] modified coalescence model in pulsed (packed and sieve plate) columns.

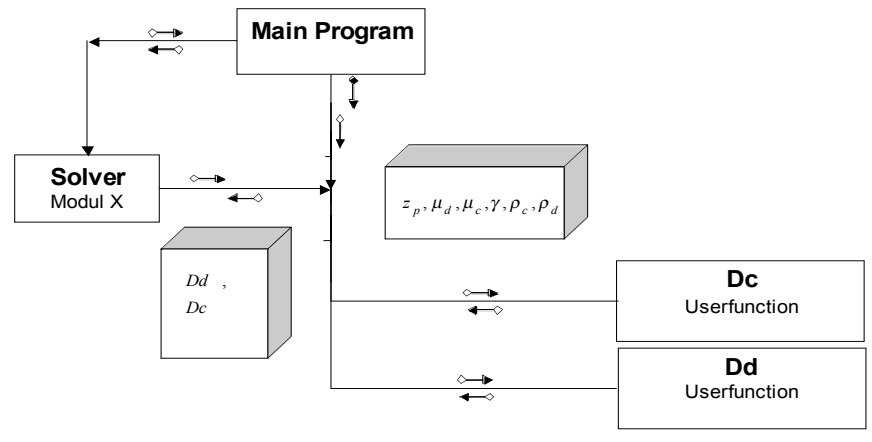

Fig. (11). Internal program structure for calculating axial dispersion coefficients.

\subsection{Axial Dispersion}

Under axial dispersion all microscopic and macroscopic mixing effects causing deviation from plug flow are summarized. The possible causes of these effects are the energy input by the pulsation or microscopic mixing as a result of turbulent flow around internals. The axial dispersion coefficient $D_{a x}$ has the same unit as the diffusion coefficient and all deviations from plug flow are summarized in this single coefficient:

$\dot{n}_{D a x, d}=-D_{a x, d} \cdot \frac{\rho_{d}}{M_{d}} \cdot \frac{\partial x}{\partial z}$

The axial dispersion is dependent on the column type and LLECMOD is calculated for the continuous and the disperse phase (see Fig. 11). The influence of the axial dispersion of the continuous phase $D_{a x, c}$ on the hydrodynamics and mass transfer in the column is greater than the influence of the axial dispersion of the disperse phase $D_{a x, d}$. The correlations for describing the axial dispersion coefficient $D_{a x, c}$ for the continuous and $D_{a x}, d$ for the disperse phase are stored in the program block user function. It is possible that the axial dispersion for the continuous phase $D_{a x, c}$ and the disperse phase $D_{a x, d}$ entered directly as a constant in the input window of the module independently of the correlations.

\section{MASS TRANSFER}

\subsection{Mass Transfer Coefficients}

The overall mass transfer coefficients (dispersed $K_{o d}$ and continuous $K_{o c}$ ) can be calculated from the individual mass transfer coefficients $k_{c}$ and $k_{d}$. The individual mass transfer coefficients mainly depend on droplet size and flow conditions inside and outside the drop. In the LLECMOD simulation program different approaches to calculate the mass transfer coefficients for the droplets inside $k_{d}$ or $k_{c}$ outside droplets can be used. In addition to traditional approaches, such as Handlos and Baron [53], Kumar and Hartland [54] and Newman [55], more recent correlations or user-defined models are included.

\subsection{Mass Transfer in Extraction Columns}

In calculating the mass transfer in spherical particles without internal circulation certainly the standard reference is Newman [55]. For the time and space dependent, the droplets concentration profile $y(r, t)$ is given in form: 
$\frac{y(r, t)-y_{l}}{y_{o}-y_{l}}=-\frac{2 R}{\pi r} \sum_{n=1}^{\infty} \frac{-1^{n}}{n} \sin \left(\frac{n \pi r}{R}\right) \exp \left(-(n \pi)^{2} F o_{d}\right)$

The result was extended later to circulating droplets [56]. Thus the droplets mean concentration $y(t)$ are calculated as:

$\frac{y(r, t)-y_{l}}{y_{o}-y_{l}}=-\frac{6}{\pi^{2}} \sum_{n=1}^{\infty} \frac{1}{n^{2}} \exp \left(-(n \pi)^{2} F o_{d}\right)$

$F o_{d}=\frac{4 D t}{d^{2}}$

$F_{\text {od }}$ is the Fourier number of the dispersed phase, $D$ is the diffusion coefficient and $t$ is the time. Instead of using series expansion for short or long periods the following approximations can also be used:

$$
\frac{y(r, t)-y_{l}}{y_{o}-y_{l}}=1-\frac{6}{\sqrt{\pi}} \sqrt{F o_{d}}+2.996 F_{d} \quad \text { for } F_{d}<0.1584
$$

$\frac{y(r, t)-y_{l}}{y_{o}-y_{l}}=\frac{6}{\pi^{2}} \exp \left(-\pi^{2} F o_{d}\right)$ for $\quad F o_{d} \geq 0.1584$

According to Kronig and Brink Eq.(46) can be extended further, when the Fourier number is formed with an effective diffusion coefficient in the form: $D_{d, \text { eff }}=B D_{d}$, the effective diffusion coefficient is given by:

$$
D_{\text {eff }}=\frac{v_{\infty} d}{2048\left(1+\mu_{d} / \mu_{c}\right)}
$$

To determine the mass transfer in this model the diffusion plays no role in $F_{\text {od }}$ in Eq. (46). This approach has been criticized by several authors because of the missing physical basis. One reason is the interfacial instability that Handlos and Baron [53] have neglected the Marangoni effects. Marangoni effect was first observed in 1865 , this effect is attributed to a change in surface tension. A generalization of the models for effective diffusion coefficient, which taken into account this instability, was proposed by Henschke and Pfennig [57]:

$$
D_{e f f}=D_{d}+\frac{v_{\infty} d}{C_{I P}\left(1+\mu_{d} / \mu_{c}\right)}
$$

The instability parameter $C_{I P}$ is adapted to the measured values. To calculate the time-dependent mean droplet concentration $D_{\text {eff }}$ in Eq.(47) is used. A further generalization of Eq.(51) is given by:

$$
\begin{aligned}
& D_{\text {eff }}=B D_{d}\left(f_{7}+C_{z i r}\left(1-f_{7}\right)\right)+\frac{v_{\infty} d}{C_{I P}\left(1+\mu_{d} / \mu_{c}\right)} \\
& f_{7}=\frac{1}{1+\left(d /\left(0.85 d_{u m}\right)\right)^{40}}
\end{aligned}
$$

The transition from rigid to circulating droplets is achieved by $f_{7}$. The use of Eq.(51) instead of Eq.(52) makes sense, if sufficient measurements are available to determine the three parameters $B, C_{z i r}$ and $C_{I P}$.

\subsubsection{Sieve Plate Geometry}

The effect of sieve trays on the mass transfer of single droplets was previously studied by Henschke [37] and the proposed extension of the model was described in Eqs.(48), (49) and (51). However, there have so far been other two effects caused by trays which are taken into account. On one hand the flow field below the perforated bottom is inhomogeneous. This is observable by a "prancing" of the droplet below the sieve plate. On the other hand the passage of droplets through the sieve holes caused an internal mixing, which leads to a change in the concentration of the boundary layer. The first effect is taken into account in the modeling by an additional amplifier factor $B_{u n}$ in Eq.(51):

$D_{\text {eff }}=D_{d}+\frac{v_{\infty} d B_{u n}}{C_{I P}\left(1+\mu_{d} / \mu_{c}\right)}$

During passage through the hole the droplet is deformed which depends on the ratio $d / d_{L}$, leading to a mixing in the drops. For a complete mixing the entire mass transfer is predictable both below and above the perforated bottom with Eq.(46). In an incomplete mixing no analytical solution exists. The concentration after passing through the hole is calculated from the concentration before passing through hole using a mixing parameter:

$$
\begin{aligned}
& \bar{y}=\left.\frac{1}{N} \sum y_{n}\right|_{\text {passes through front hole }} \\
& \left.y_{n}\right|_{\text {passes through front hole }}=\Omega \cdot \bar{y}+\left.(1-\Omega) y_{n}\right|_{\text {passes through front hole }}
\end{aligned}
$$

In the numerical calculations, the droplet volume is divided into $N$ elements, each with a radius $r_{n}$. The mass flow passing from element to element can be calculated over each time interval at a time $t$, according to Eq.(57).

$$
\Delta \dot{m}(t)=A_{n-1} \rho_{d} D_{e f f} \frac{y_{n-1}(t)-y_{n}(t)}{r_{n-1}-r_{n}}
$$

The calculation of the new concentrations of the elements is in accordance with Eq. (58):

$$
y_{n}(t-\Delta t)=y_{n}(t)+\frac{\left[\Delta \dot{m}_{n}(t)-\Delta \dot{m}_{n+1}(t)\right] \Delta t}{\rho_{d} \Delta V}
$$

For the numerical calculations the time interval $\Delta t$ and the number of elements $N$ are given. The values of $C_{I P}, B_{U N}$ and $\Omega$ which must be specified in the model, as a result from a fit to the measured values. The empirical dependencies of these parameters are given by:

$$
\begin{aligned}
& \Omega=\exp \left(a_{19} \frac{d_{L}}{d}\right) \text { with } a_{19}<0 \\
& B_{u n}=\frac{1.582}{\phi^{a_{20}}}\left[1-\exp \left(-\frac{v_{L}}{v_{\infty}}\right)\right]
\end{aligned}
$$


They are based on results for a series of sieves with different geometries. In the development of the two above equations a better description of the real physical behavior is taken into consideration. The term $\phi^{a_{20}}$ in the denominator takes into account the increasing inhomogeneity of the flow with decreasing the relative opening area. Nevertheless, to allow a rough column simulation, a simplified mass transfer model with $\Omega=0$ (no mixing within the drops) is introduced:

$$
D_{\text {eff }}=D_{d}+\frac{v_{\infty} d}{C_{I P} \phi^{a_{20}}\left(1+\mu_{d} / \mu_{c}\right)}
$$

The simple relationship $B_{u n}=1 / \Phi^{a 20}$ is used. The two remaining parameters for $C_{I P}$ and $a_{20}$ are determined for water-acetone-toluene (w-a-t) system to be $C_{I P}=5527$ and $a_{20}=$ 0.734 , while for the system water-acetone-butyl acetate (w-ab) $C_{I P}=4361$ and $a_{20}=0.329$ [37].

\subsubsection{Structured Packing Geometry}

The same mass transfer model used for pulsed columns with sieve plate is applied for columns with structured packing.

The hydrodynamic parameters like droplet velocity, axial back mixing, coalescence and breakage characterize the flow and mass transfer influence in addition to the description of the separation and column efficiency. The required relations for the prediction can be obtained either by experiment or from the available literature. The available correlations, identified by their sources or authors are listed in Table 4.

\section{TRANSIENT ANALYSIS}

An understanding of the transient behavior of chemical processes is important from both process design and process control perspectives. In LLECMOD the column transient behavior can be analyzed with a change in the concentration of the disperse phase $C_{d}$, concentration of the continuous phase $C_{c}$, agitator speed $N$, flow rate of disperse phase $Q_{d}$ and flow rate of continuous phase $Q_{c}$.

The simulation results are: holdup $\Phi(z)$, transition concentration of the continuous phase $C_{c}(z)$ and the disperse

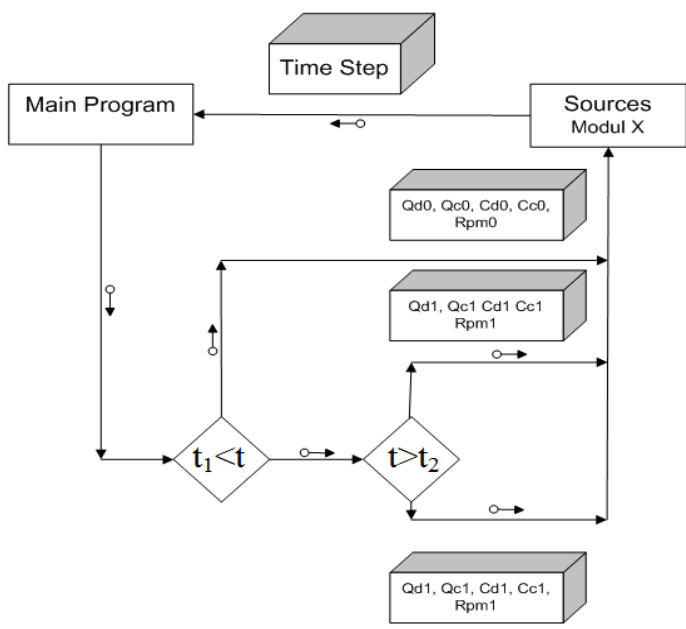

Fig. (12). Internal program structure for transient analysis.

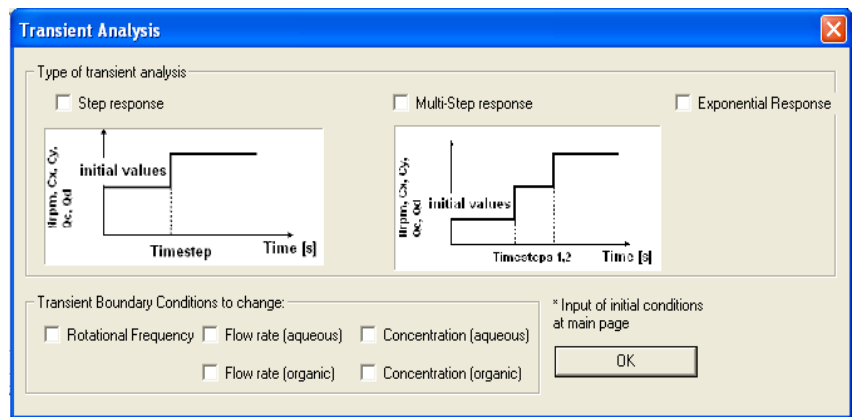

Fig. (13). Input mask for transient analysis (I).

phase $C_{d}(z)$, Sauter diameter $d_{32}(z)$ and droplet size distribution along the column height. The abrupt change of any of that input variables can be specified in the form of one or two step functions or as exponential function. Also, a Dirac pulse function can be created with a sudden change and an immediate equal counter-action.

Fig. (12) shows the algorithm for the calculation of time dependent input variables. A run of the solution algorithm is associated with a time step. The current time step is the criterion for changing the input variables for the next time step. The time of change for each input variable can be used

Table 4. Available Hydrodynamics and Mass Transfer Correlations Implemented in LLECMOD

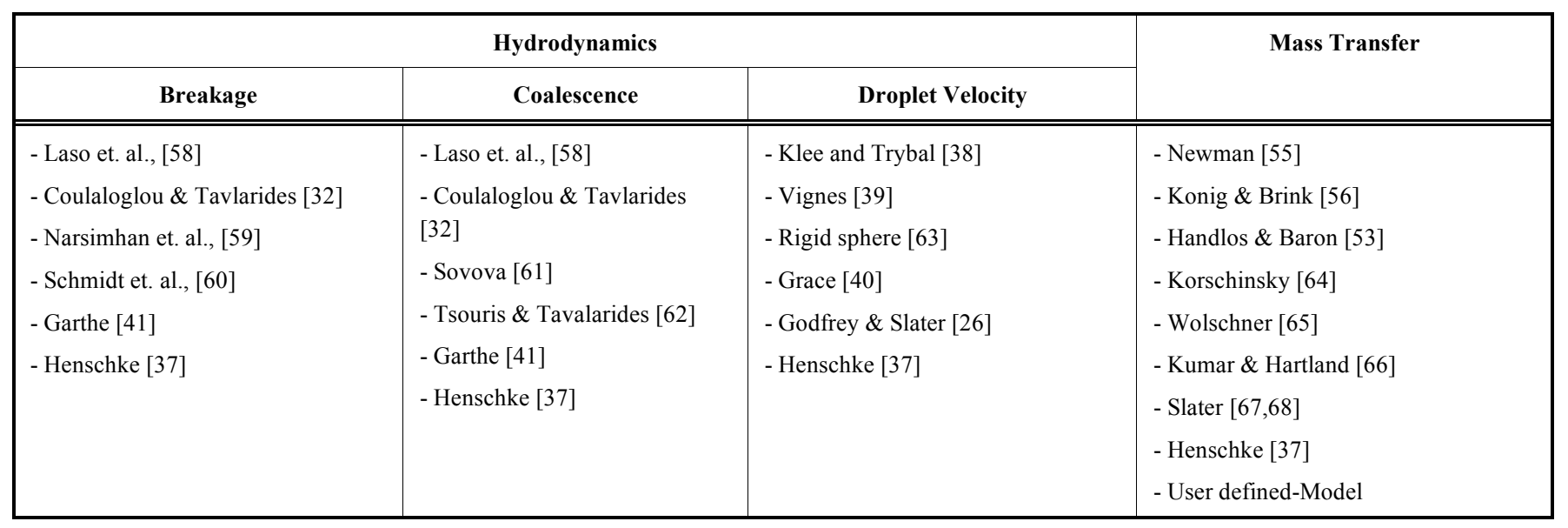




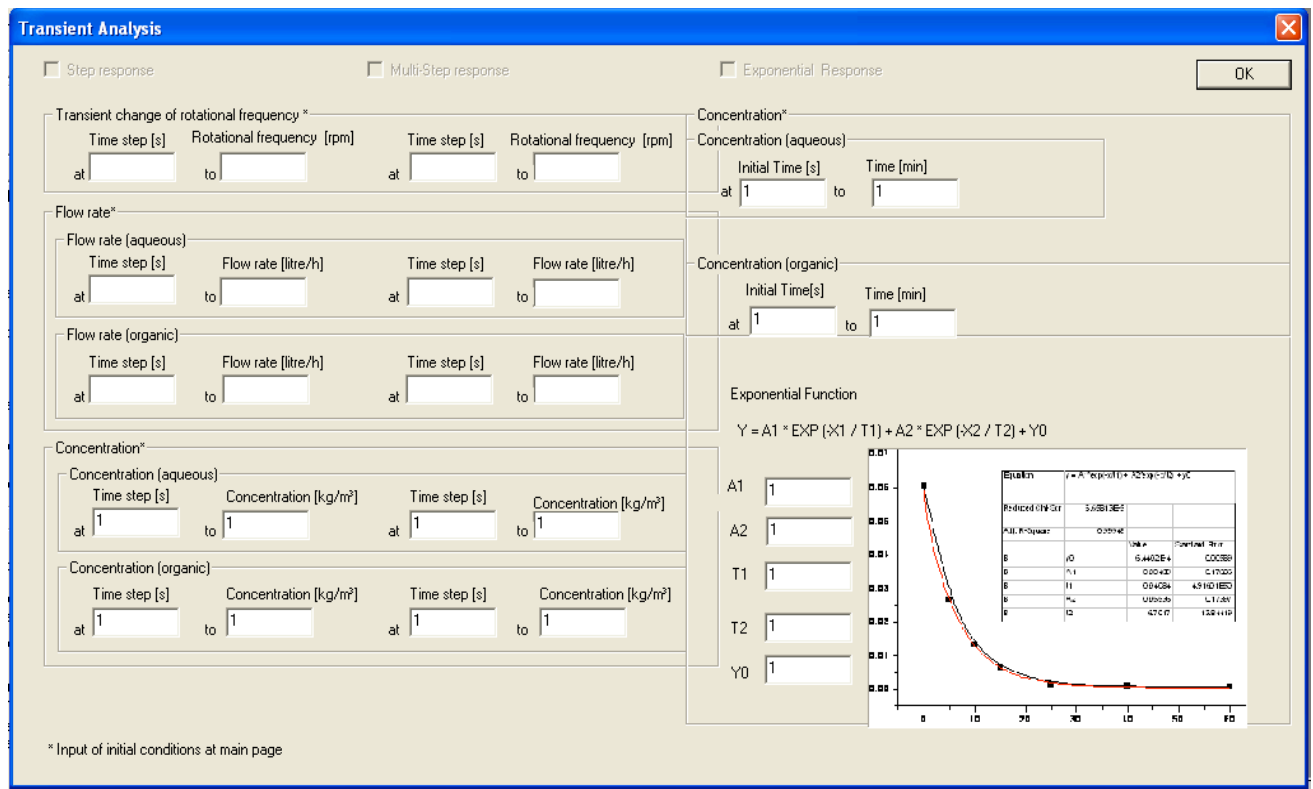

Fig. (14). Input mask for transient analysis (II).

freely. The rate of change can take place in one or two steps. In the input screen there is a possibility to select single or multi-step-selected response.

Fig. (13) shows the dialog box to select the number of changes or to change the values. The simulation parameters from the beginning of the simulation up to the time step of the changes correspond to the input in the implementation of non-transient analysis. The confirmation of the input leads to a second input mask, which is shown in Fig. (13). Here the time step is initialized, from which the change in the predetermined input variables start to take effect. In addition to the time step, the value of the changing size for the appropriate time step is entered. In the dialog box which is shown in Fig. (14), three choices are available for the transient simulation. The first one is a step function, the second one is the multistep function and the third one is the exponential function. The latter is given by:

$y=a_{1} \exp \left(\frac{-x_{1}}{t_{1}}\right)+a_{2} \exp \left(\frac{-x_{2}}{t_{2}}\right)+y_{o}$

The coefficients $a_{1}, a_{2}, t_{1}, t_{2}$ and $y_{o}$ are determined by fitting the experiential data to this function Eq. (62).

\section{RESULTS}

The simulation of the sieve plate and packed extraction columns are carried out using the modified version of LLECMOD with the recently added correlations for mass transfer, droplet velocity as well as the breakage and coalescence frequencies that can be selected via the general user interface input window for LLECMOD (see Fig. 15). The simulation can be carried out for any desired column type (packed, sieve plate, Kühni and Rotating Disk Contactor). Also, this figure shows all the input parameter necessary for the simulation and the column geometry used, which is given in Table 5. The two recommended EFCE test systems, namely water-acetone-toluene (w-a-t) and water-acetone- butyl acetate (w-a-b) and an industrial test system have been used in the simulation for pulsed packed and sieve plate extraction columns. All the simulations were carried out under the conditions of mass transfer direction from the continuous to the dispersed phase. The continuous phase volumetric flow rate $\left(Q_{c}\right)=40 \mathrm{l} / \mathrm{hr}$ and the dispersed phase volumetric flow rate $\left(Q_{d}\right)=48 \mathrm{l} / \mathrm{hr}$. The inlet feed distribution is considered as normal feed distribution. All the experimental data for the EFCE test systems are from Garthe [41]. Garthe [41] investigated the fluiddynamics and mass transfer behavior of droplet swarms in the pulsed extractor as illustrated in Fig. (16). Pulsed experiments were carried out in the extractor with different types of column internals such as sieve trays and structured packings. The size distribution of the drops was measured at three positions within the active part of the column by photoelectrical suction probes [41]. The holdup within the active part of the column was determined using two slide valves [41]. The concentration of the solute in the continuous and the dispersed phase was determined at the phase inlets and outlets as well as at three positions within the column [41].

Table 5. Column Geometry

\begin{tabular}{|l|l|}
\hline \multicolumn{1}{|c|}{ Dimension } & \multicolumn{1}{c|}{ Value } \\
\hline \hline Height of the column $(H)$ & $4.40(\mathrm{~m})$ \\
\hline Inlet for the continuous phase $\left(Z_{c}\right)$ & $3.80(\mathrm{~m})$ \\
\hline Inlet for the dispersed phase $\left(Z_{d}\right)$ & $0.85(\mathrm{~m})$ \\
\hline Column diameter $\left(d_{c}\right)$ & $0.08(\mathrm{~m})$ \\
\hline
\end{tabular}

The following recently implemented models and correlations are used in the simulation: The velocity model is Henschke [37], where the model parameters used in the simulations are given in Table $\mathbf{6}$. The breakage frequency model is after Garthe [41] and the model parameters used in the simu- 


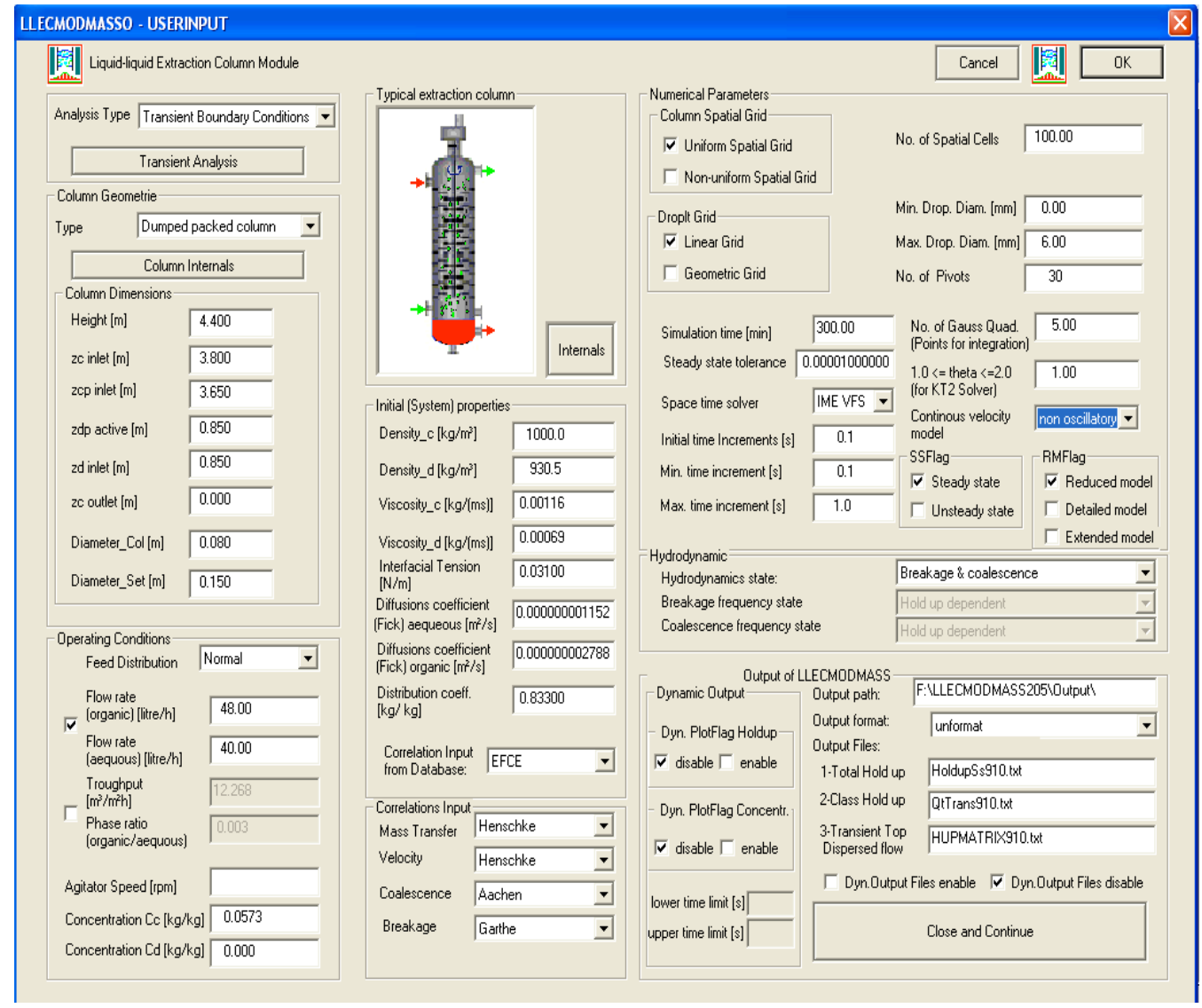

Fig. (15). LLECMOD general user interface.

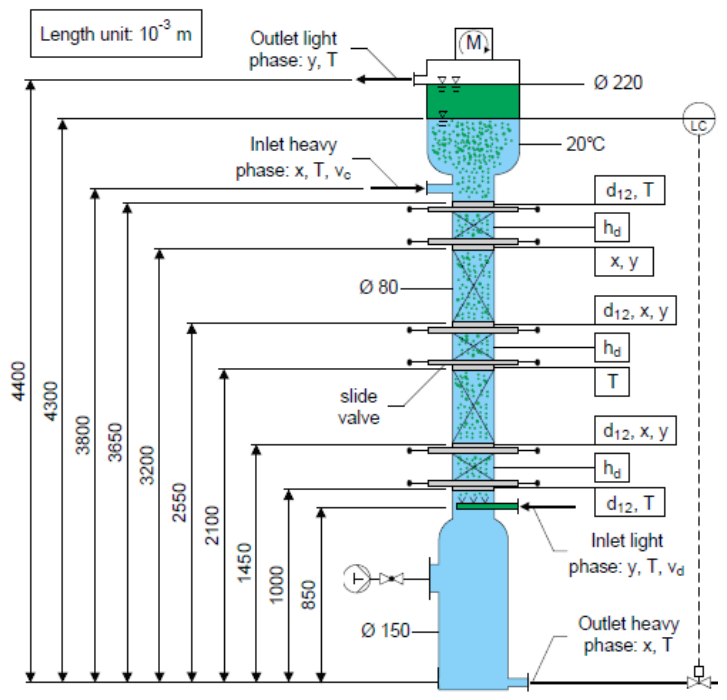

Fig. (16). Pilot plant extraction column used in the experimental study of Garthe [41].

lations are given in Tables $\mathbf{1}$ and $\mathbf{2}$. The coalescence frequency model is Henschke [37] model and the correspondence parameters used in the simulations are given in Tables 3 and 6. The mass transfer model is after Henschke [37]. The model parameters used in the simulations are given in Table 6. These correlations and models were found the best for the simulation of pulsed (packed and sieve plate) extraction columns. These models and correlations cover a wide range of operating conditions and physical properties of test system varies from the low interfacial tension test systems to the high interfacial tension test systems. However, the other correlations given in literature are very limited to special cases of operating conditions and the physical properties.

\subsection{Pulsed Packed Columns}

Fig. (17) shows the LLECMOD input dialogue for pulsed packed column, like pulsation intensity and the packing's internal geometry, which are documented in Table 2, Table 7 and Table $\mathbf{8}$. 
Table 6. Simulation Parameters

\begin{tabular}{|l|l|l|l|l|}
\hline \multirow{2}{*}{ Parameter } & \multicolumn{2}{c|}{ Test System } & \multicolumn{1}{c|}{ Industrial System } & Water-MIBK \\
\cline { 2 - 6 } & \multicolumn{1}{|c|}{ w-a-b } & \multicolumn{1}{c|}{ w-a-t } & \multicolumn{2}{c|}{} \\
\hline \hline $\mathrm{C}_{\mathrm{IP}}(-)$ & 9720 & 9445 & 14800 & - \\
\hline $\mathrm{d}_{\mathrm{um}}(\mathrm{mm})$ & 3.71 & 7.1 & 2.32 & 3.71 \\
\hline $\mathrm{a}_{15}(-)$ & 2.246 & 1.52 & 3.35 & 2.246 \\
\hline $\mathrm{a}_{16}(-)$ & 1.779 & 4.5 & 1.166 & 1.779 \\
\hline$\alpha_{\mathrm{um}}(-)$ & 8 & 8 & 8.0 & 8 \\
\hline$\alpha_{\mathrm{gr}}(-)$ & 8 & 8 & 8.0 & 8 \\
\hline$\zeta_{8}(-)$ & 1500 & 2500 & 2500 & 1500 \\
\hline $\mathrm{C}(-)$ & 102.0 & 69.4 & 253.7 & 47.2 \\
\hline
\end{tabular}

\subsubsection{The Water-acetone-toluene Test System}

Fig. (18) draws a comparison between the simulated and experimental Sauter mean diameter profiles and Fig. (19) shows the simulated and experimental holdup profile of the disperse phase along the pulsed packed column height. Fig. (20) depicts the simulated and experimental solute concentration profiles in the continuous and dispersed phases along the pulsed packed column height.

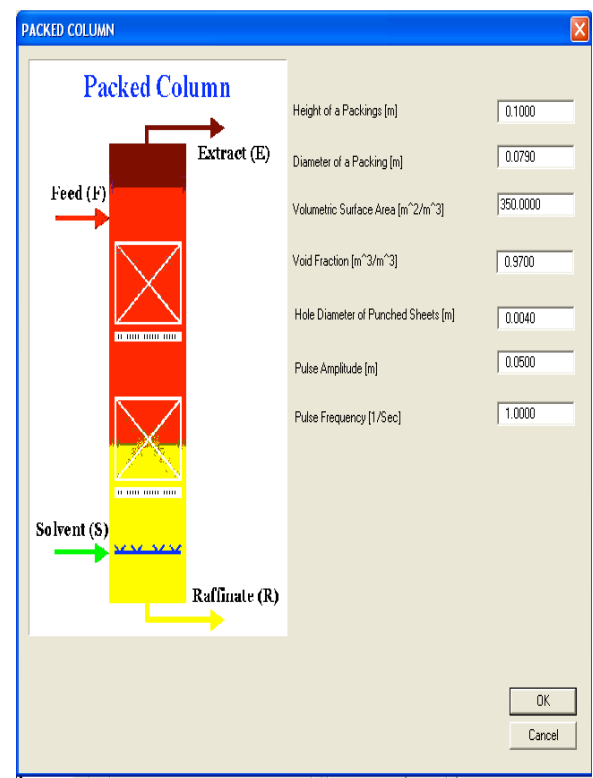

Fig. (17). Input dialogue for pulsed packed column internal geometry.

Table 7. Geometrical Data of Structured Packings (MontzPak B1-350)

\begin{tabular}{|l|l|}
\hline Diameter of a packing $\left(d_{P}\right)$ & $79(\mathrm{~mm})$ \\
\hline Volumetric surface area $\left(a_{P}\right)$ & $350\left(\mathrm{~m}^{2} \cdot \mathrm{m}^{-3}\right)$ \\
\hline void fraction $\left(\varphi_{P}\right)$ & $0.90\left(\mathrm{~m}^{3} \cdot \mathrm{m}^{-3}\right)$ \\
\hline height of a packing $\left(h_{P k}\right)$ & $100(\mathrm{~mm})$ \\
\hline hole diameter of punched sheets $\left(\mathrm{dh}_{P}\right)$ & $3(\mathrm{~mm})$ \\
\hline
\end{tabular}

\subsubsection{The Water-acetone-butyl Acetate Test System}

Fig. (21) shows the comparison between the simulated and experimental Sauter mean diameter profiles for the pulsed packed column; the discrepancy of the simulated experimental results from those obtained in the experiment is almost negligible. Fig. (22) depicts the holdup profile of the disperse phase for the pulsed packed column compared to the experimental results from those obtained in the experi-

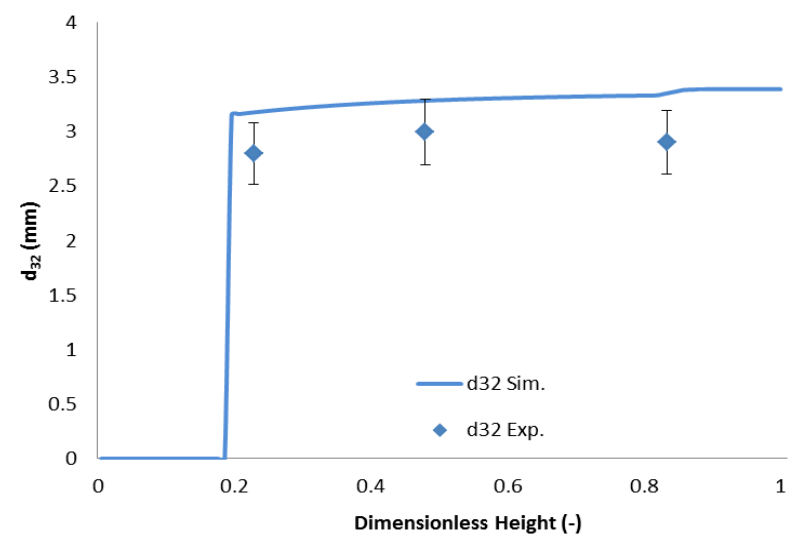

Fig. (18). Simulated mean droplet diameter compared to the experimental results [41] in pulsed packed column.

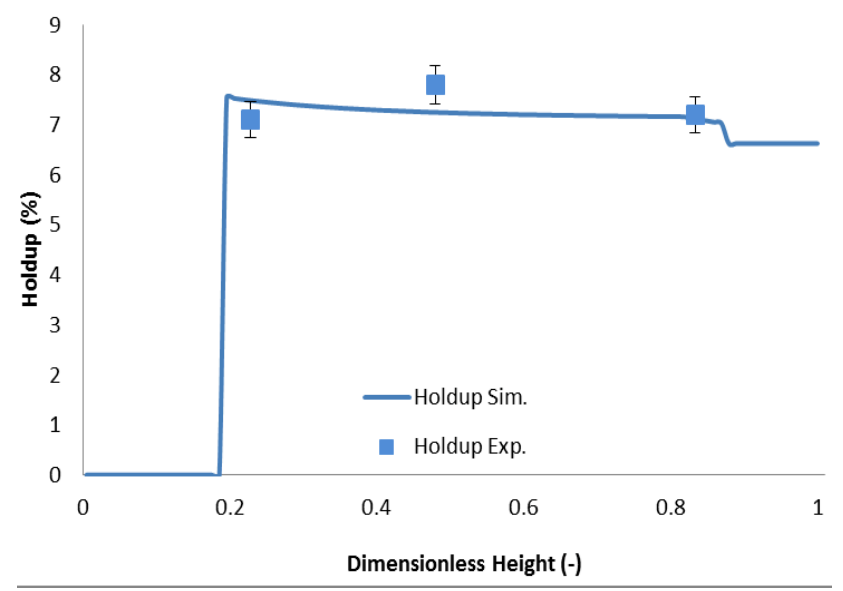

Fig. (19). Simulated holdup compared to the experimental results [41] in pulsed packed column. 
Table 8. Characteristic Droplet Diameter $d_{\text {stab }} \& d_{100}$ in Pulsed Packed Columns

\begin{tabular}{|l|l|l|l|}
\hline \multicolumn{1}{|c|}{ Test System } & \multicolumn{1}{c|}{$\boldsymbol{a . f}\left(\mathbf{c m . s ^ { - 1 } )}\right.$} & \multicolumn{1}{c|}{$\boldsymbol{d}_{\text {stab }}(\mathbf{m m})$} & $\boldsymbol{d}_{100}(\mathbf{m m})$ \\
\hline \hline water-acetone-toluene & 1.0 & 3.3 & 7.5 \\
\hline water-acetone-butyl acetate & 1.0 & 2.2 & 4.0 \\
\hline Industrial test system & 1.0 & 4.0 & 2.2 \\
\hline
\end{tabular}

ment, a good agreement was achieved. Fig. (23) shows the concentration profile for both phases along the pulsed packed column height. Comparison between the experimental and calculated concentration profiles over the total column-height shows a good agreement.

\subsubsection{Industrial Test System: Hydrodynamics and Mass Transfer Simulation Results}

The simulation program LLECMOD has validated with a pulsed packed column in an industrial environment. The physical properties of the industrial test system are given in Table 9. The extraction factor was 0.8963 and extraction efficiency of $95 \%$ was required.

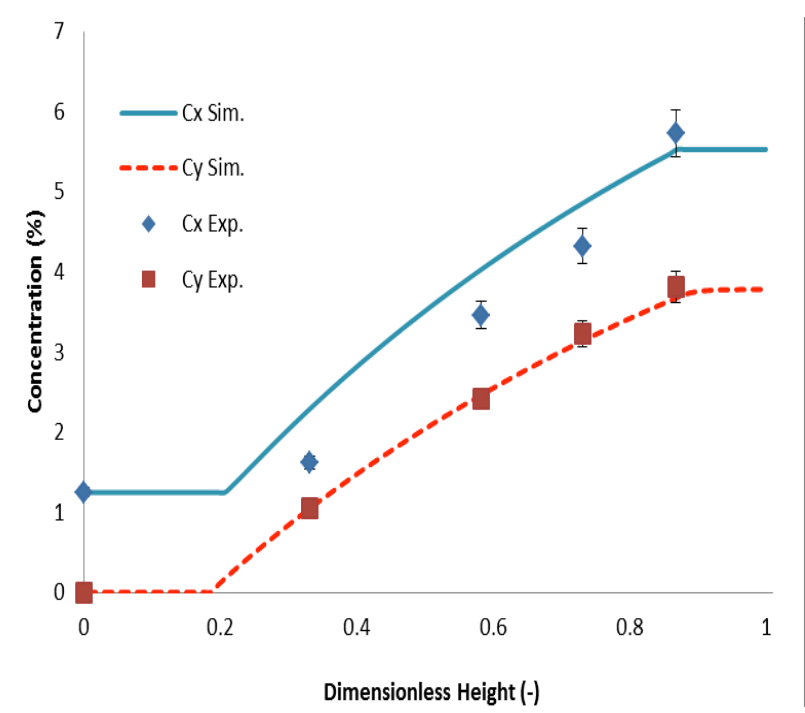

Fig. (20). Simulated concentration profiles in both phases compared to the experimental results[41] in pulsed packed column.

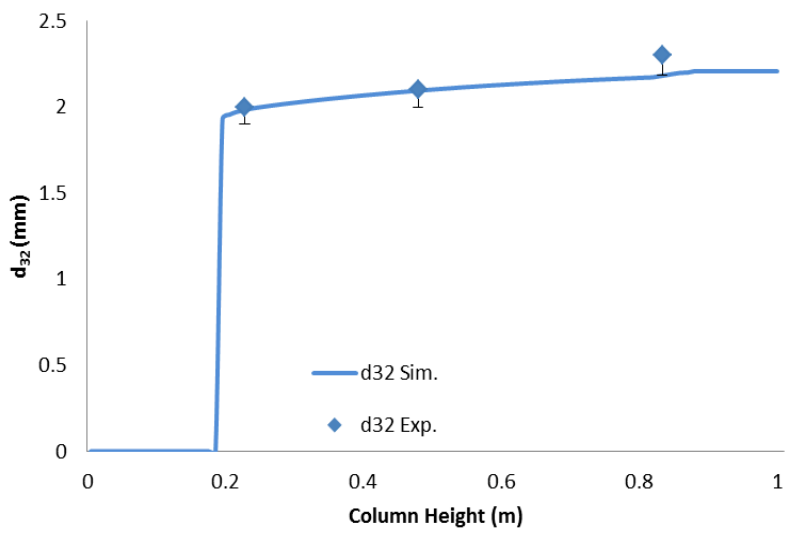

Fig. (21). Simulated mean droplet diameter compared to the experimental results [41] in pulsed packed column.
Fig. (24) gives the evaluation of the simulated hydrodynamics profiles (Sauter mean diameter and holdup) along the column height for the industrial test system in a pulsed packed column. Fig. (25) shows the concentration profile for both phases along the column height in a pulsed packed column. Comparison between the experimental and calculated concentration profiles over the total column-height shows a good agreement.

\subsection{Pulsed Sieve Plate Column}

Tables 1, $\mathbf{1 0}$ and $\mathbf{1 1}$ give the system parameters for sieve plate column. In Table $\mathbf{1 0}$ are the geometrical data of used internals of the sieve plate, which is installed inside the active part of the columns and used in the simulation. Fig. (26) depicts the LLECMOD input frame of the internal geometry of pulsed sieve plate column.

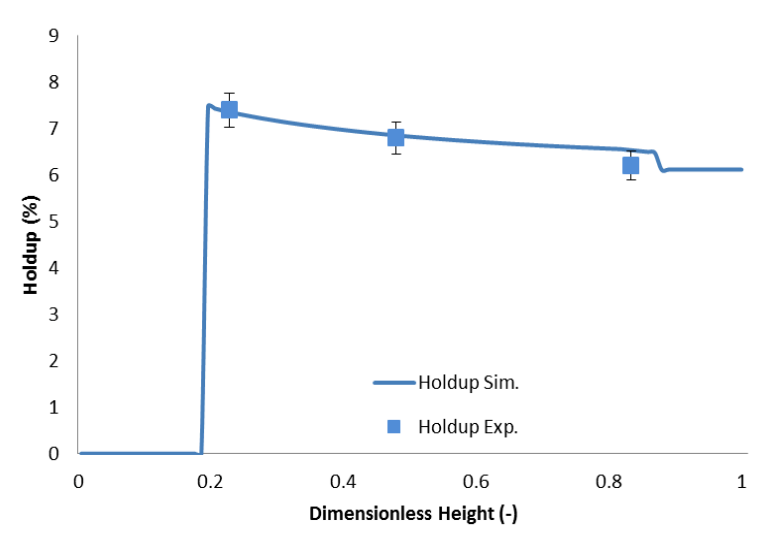

Fig. (22). Simulated holdup compared to the experimental results[41] in pulsed packed column.

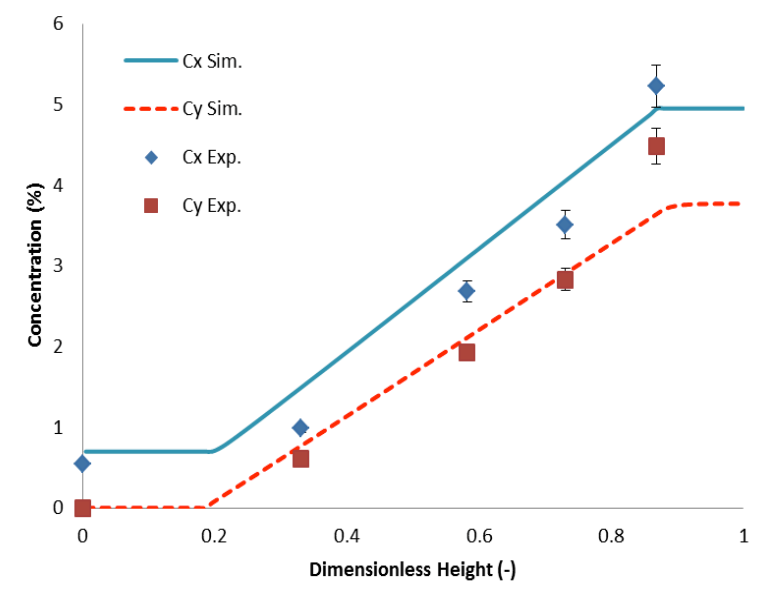

Fig. (23). Simulated concentration profiles in both phases compared to the experimental results [41] in pulsed packed column. 
Table 9. Normalized Physical Properties of BASF Technical System

\begin{tabular}{|l|l|l|l|l|l|l|l|}
\hline & \multicolumn{1}{|c|}{$\boldsymbol{x}_{\text {Raffinate }}(-)$} & \multicolumn{1}{|c|}{$\boldsymbol{y}_{\text {Extract }}(-)$} & \multicolumn{1}{|c|}{$\boldsymbol{\rho}_{\boldsymbol{y}}(-)$} & \multicolumn{1}{|c|}{$\boldsymbol{\rho}_{\boldsymbol{x}}(-)$} & \multicolumn{1}{c|}{$\boldsymbol{\mu}_{\boldsymbol{y}}(-)$} & $\boldsymbol{\mu}_{x}(-)$ & \multicolumn{1}{c|}{$\boldsymbol{\sigma}(-)$} \\
\hline \hline initial & 0.9922 & 1.0 & 0.9954 & 0.9261 & 0.902 & 0.162 & 0.591 \\
\hline final & 0.0806 & 0.0623 & 1.0 & 0.9197 & 1.0 & 0.144 & 1.0 \\
\hline
\end{tabular}

\subsubsection{The Water-acetone-toluene Test System}

Fig. (27) draws the comparison between the simulated and experimental Sauter mean diameter profiles for the sieve plate column; a relatively good agreement was achieved. Fig. (28) shows the holdup profile of the disperse phase in a pulsed sieve plate column compared to the experimental results from those obtained in the experiment, again good agreement was achieved. Fig. (29) depicts the simulated and experimental solute concentration profiles in the continuous and dispersed phases along the pulsed sieve plate column height. The relative error is less than $5 \%$.

\subsubsection{The Water-acetone-butyl Acetate Test System}

Fig. (30) shows the comparison between the simulated and experimental Sauter mean diameter profiles for the sieve plate column; the discrepancy of the simulated experimental results from those obtained in the experiment is almost negligible except at the outlet of the column. Fig. (31) depicts the holdup profile of the dispersed phase in a pulsed sieve plate column compared to the experimental results from

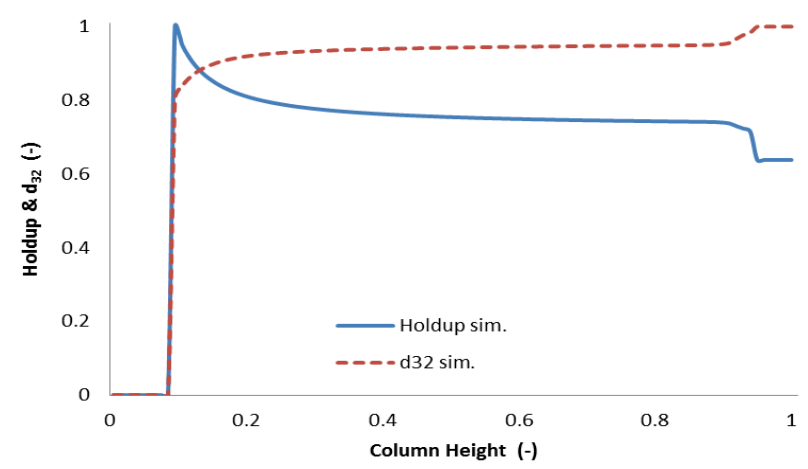

Fig. (24). Simulated holdup and mean droplet diameter profiles in industrial pulsed packed column.

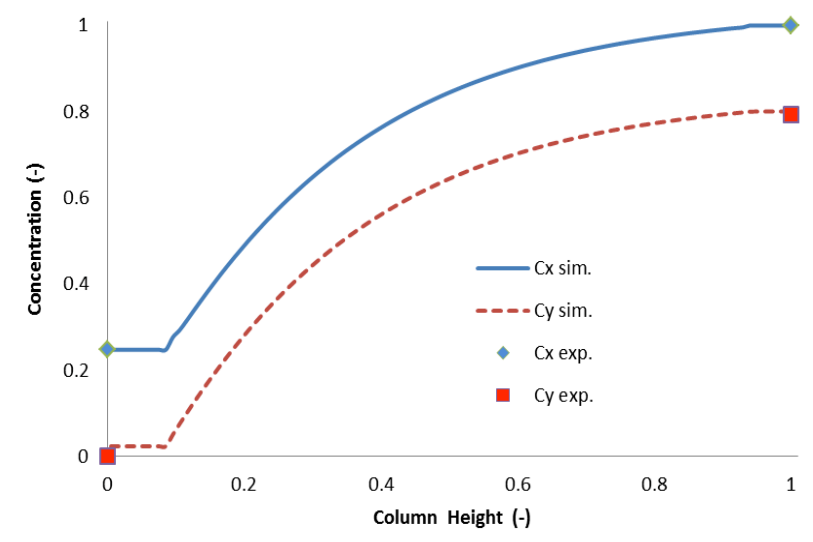

Fig. (25). Simulated concentration profiles in both phases compared to the experimental results in industrial pulsed packed column. those obtained in the experiment, a good agreement was achieved. Fig. (32) depicts the simulated and experimental solute concentration profiles in both phases along the pulsed

\subsection{Dynamics Results}

\subsubsection{Water-acetone-toluene System}

The transient analysis of columns allows the study of the effect of disturbances or specific changes in the operating conditions on the column behavior. The results for the dynamic behavior of a pulsed packed extraction column for an abrupt change of the flow rate of disperse phase and its effect on holdup is studied here for water-acetone-toluene (wa-t) test system. The initial volumetric flow rate is $48 \mathrm{l} / \mathrm{h}$ for disperse phase at a time of $750 \mathrm{sec}$ after reaching a steadystate operation; the inflow of the disperse phase is increased to $68 \mathrm{l} / \mathrm{h}$ as shown in Fig. (33). However, Fig. (34) shows the

Table 10. Geometrical Data of Pulsed Sieve Tray

\begin{tabular}{|l|l|}
\hline Diameter of sieve tray $\left(d_{s t}\right)$ & $79(\mathrm{~mm})$ \\
\hline Diameter of sieve try holes $\left(d_{h}\right)$ & $2(\mathrm{~mm})$ \\
\hline Relative free cross-sectional area $\left(\varphi_{s t}\right)$ & $0.2\left(\mathrm{~m}^{2} \cdot \mathrm{m}^{-2}\right)$ \\
\hline Height of compartment $\left(h_{s t}\right)$ & $100(\mathrm{~mm})$ \\
\hline
\end{tabular}

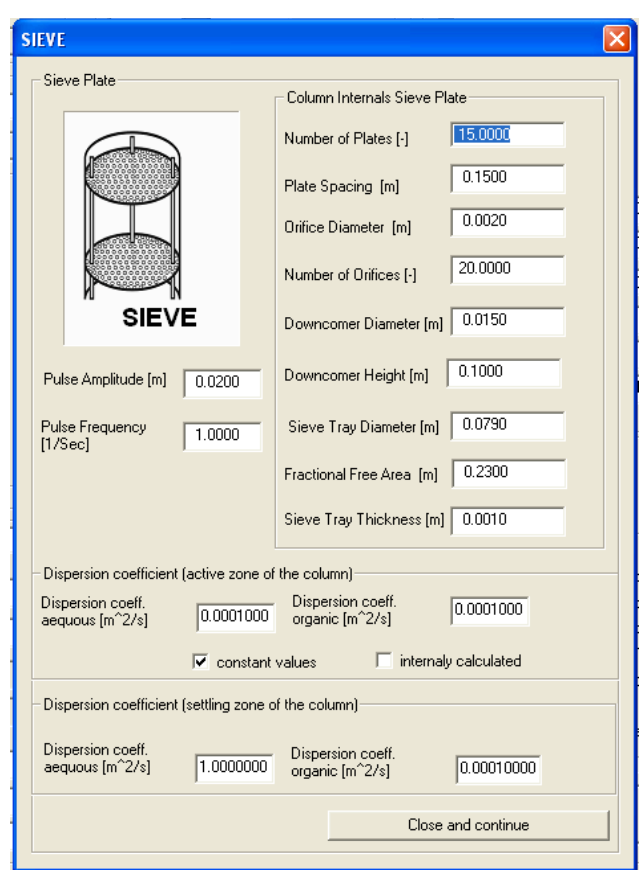

Fig. (26). Input dialogue for pulsed sieve plate column internal geometry. 


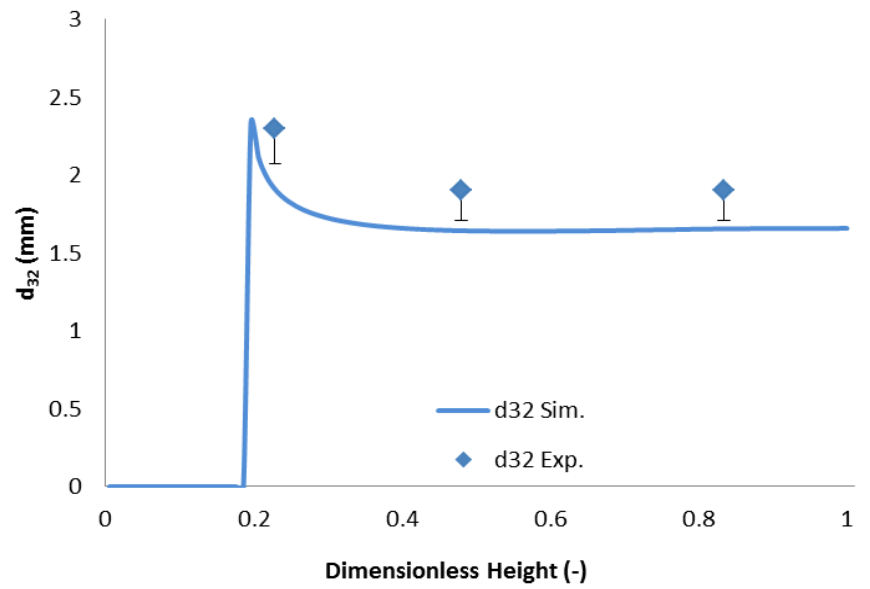

Fig. (27). Simulated mean droplet diameter compared to the experimental results[41] in pulsed sieve plate column.

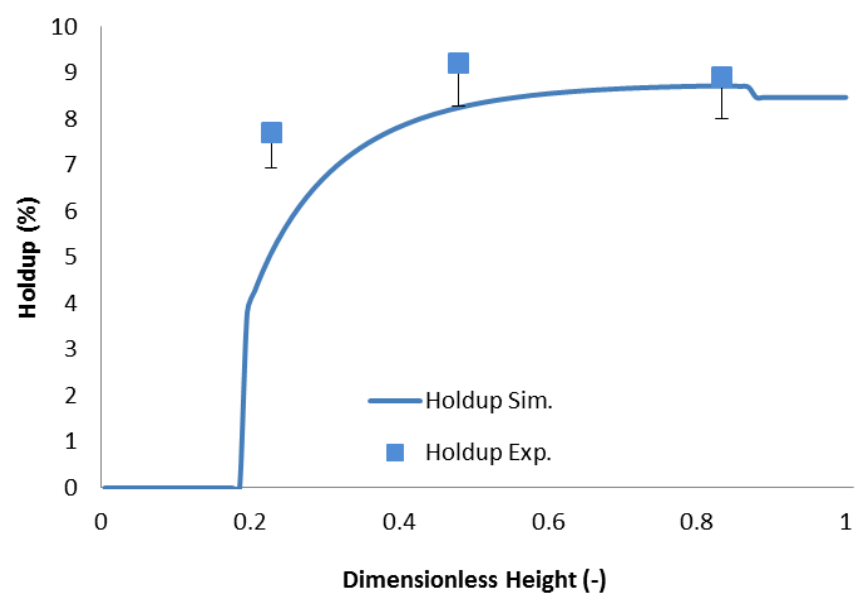

Fig. (28). Simulated holdup compared to the experimental results[41] in pulsed sieve plate column.

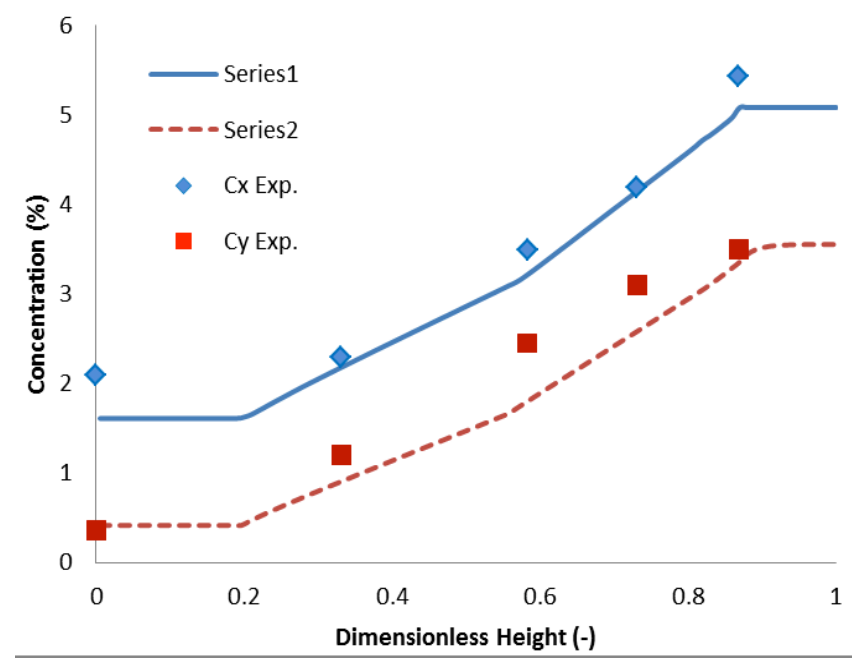

Fig. (29). Simulated concentration profiles in both phases compared to the experimental results[41] in pulsed sieve plate column.

dynamic response of the holdup dispersed phase profile due to a step change of the volumetric flow rate from $48 \mathrm{l} / \mathrm{h}$ to 68 $l / h$ which is reflected in an increase in holdup immediately after the time of the change in the simulation at $750 \mathrm{sec}$.

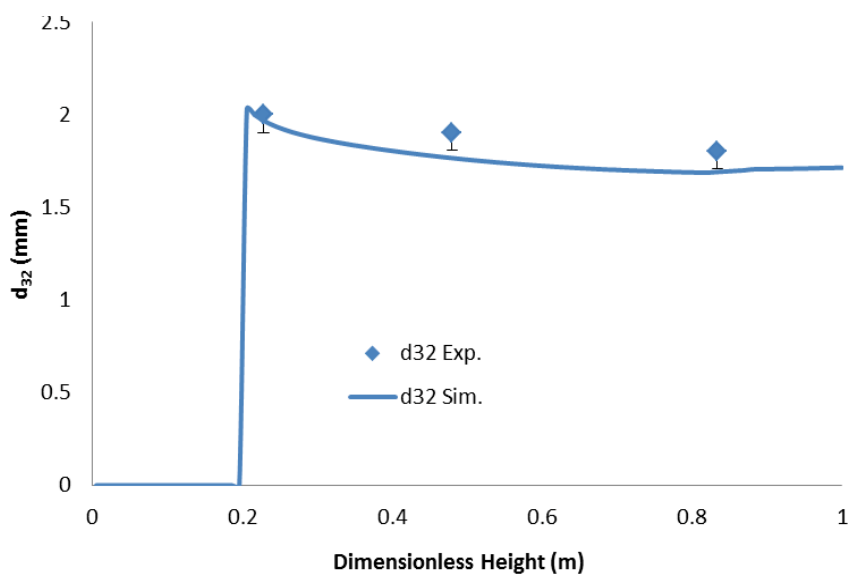

Fig. (30). Simulated mean droplet diameter compared to the experimental results[41] in pulsed sieve plate column.

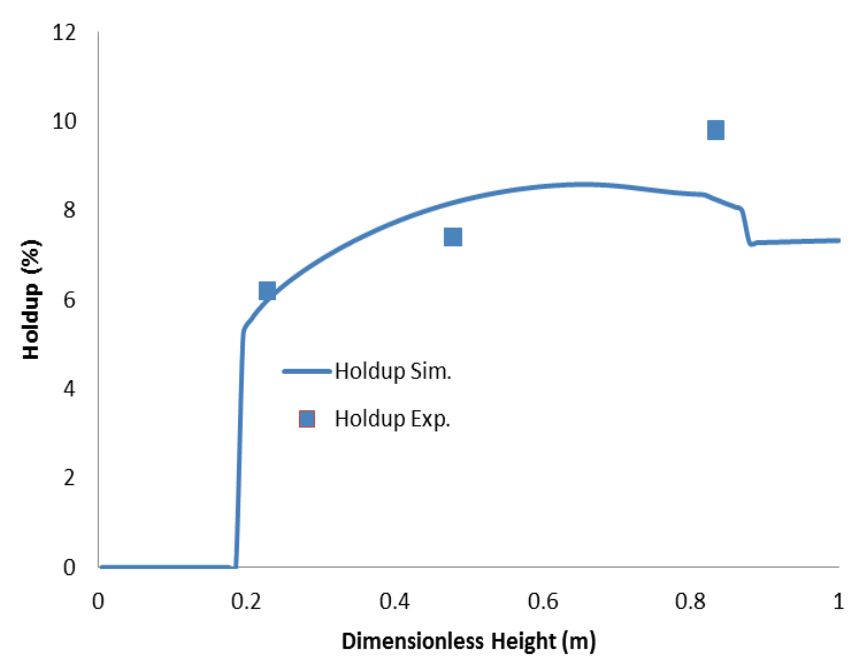

Fig. (31). Simulated holdup compared to the experimental results[41] in pulsed sieve plate column.

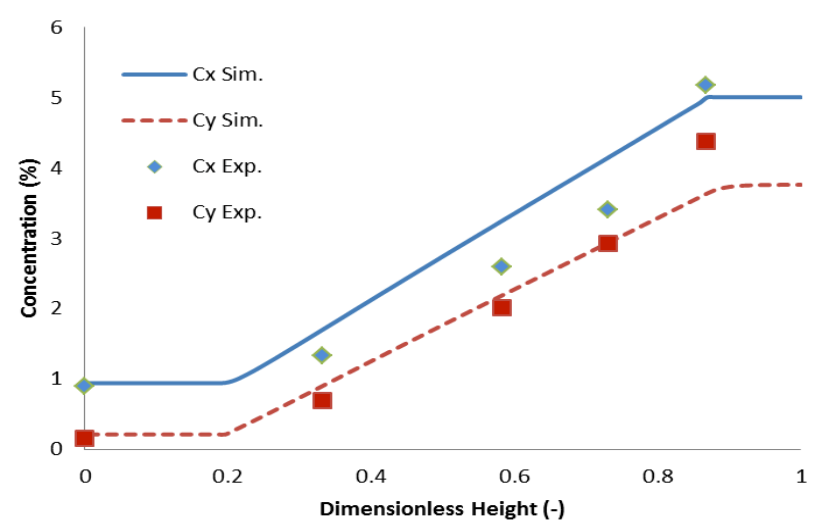

Fig. (32). Simulated concentration profiles in both phases compared to the experimental results[41] in pulsed sieve plate column.

\subsubsection{Water- methyl Isobutyl Ketone System}

Fig. (35) depicts the validation of LLECMOD transient simulation, the simulation transient results compared to the experimental data. The simulation results agree with the experimental data fairly well. The test system used here is water- methyl isobutyl ketone (w-MIBK); the physical proper- 
Table 11. Characteristic Droplet Diameter $d_{\text {stab }} \& d_{100}$ in Pulsed Sieve Plate Column

\begin{tabular}{|c|c|c|c|c|}
\hline Test System & $d_{h}(m m)$ & a. $f\left(\mathrm{~cm} \cdot \mathrm{s}^{-1}\right)$ & $d_{\text {stab }}(\mathrm{mm})$ & $d_{100}(m m)$ \\
\hline water-acetone-toluene & $2 \mathrm{~mm}$ & 1.50 & 1.7 & 4.1 \\
\hline water-acetone-butyl acetate & $2 \mathrm{~mm}$ & 1.00 & 2.0 & 3.8 \\
\hline water-MIBK & $3.2 \mathrm{~mm}$ & 5.34 & 1.0 & 2.5 \\
\hline
\end{tabular}

ties of the test system are given in Table 12. This figure shows the dynamic evolution of dispersed phase holdup due to a positive step change in the dispersed phase volumetric flow rate. The operating conditions and the column geometry used in this dynamic simulation are given in Tables $\mathbf{1 3}$ and 14 respectively.

\section{CONCLUSIONS}

In this work two pulsed extraction column modules (namely: pulsed packed and pulsed sieve plate column) are integrated into the LLECMOD program for the simulation of coupled hydrodynamics and mass transfer for liquid-liquid extraction columns. The user-friendly input dialogs and the

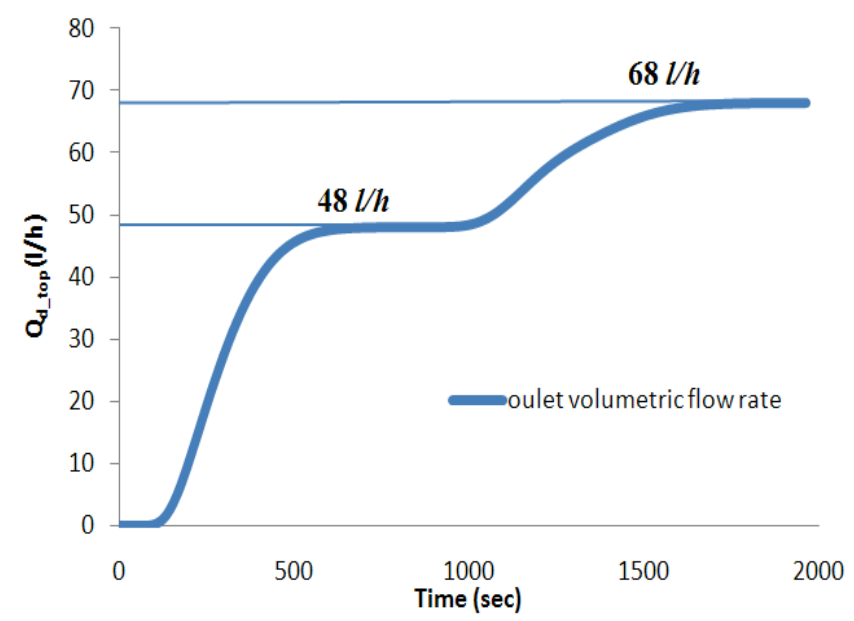

Fig. (33). Dynamic evolution of dispersed phase outlet flow rate in pulsed packed column.

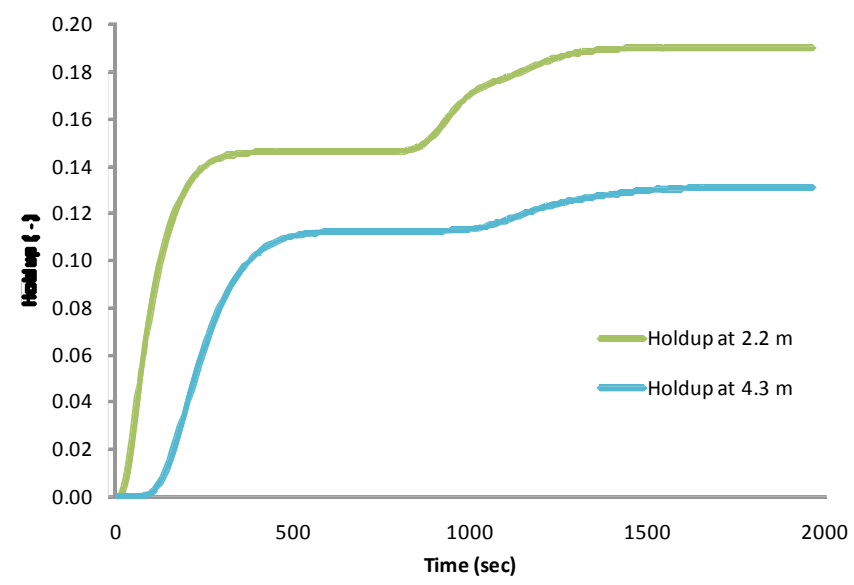

Fig. (34). Dynamic evolution of holdup profile in pulsed packed column. user functions input modules make the program very general and simple to use. The LLECMOD non-equilibrium bivariate (in solute concentration and droplet diameter) population balance model is found capable of simulating new types of extraction columns; namely, pulsed and un-pulsed (packed and sieve tray) columns in addition to the stirred ones (Kühni \& RDC types). The steady state column hydrodynamics can be efficiently predicted by using the correlations discussed, the values of the parameters in these correlations are available in the literature for the standard test systems. However, for technical systems the steady state column hydrodynamics can be efficiently predicted by only adjusting few parameters in the droplet coalescence model, in the breakage model, droplet velocity and in mass transfer model. The parameters can be obtained from available experimental data, small labscale experiments or default values of LLECMOD can be used. The steady state performance of pulsed (sieve plate and packed) extraction column are studied using a detailed population balance framework as an alternative to the commonly applied dispersion and backmixing models. The simulated holdup, mean droplet diameter and the mass transfer profiles for both test systems (t-a-w \& b-a-w) in pulsed (packed and sieve plate) extraction columns has extensively verified with EFCE test systems with very good agreement. Moreover, LLECMOD was successfully validated against industrial extraction column data and even transient analysis gave no problems. In general, LLECMOD provides a useful tool for the scale up and simulation of agitated liquid extraction columns considering a more realistic conception of the dispersed phase discrete nature.

\section{CONFLICT OF INTEREST}

None declared.

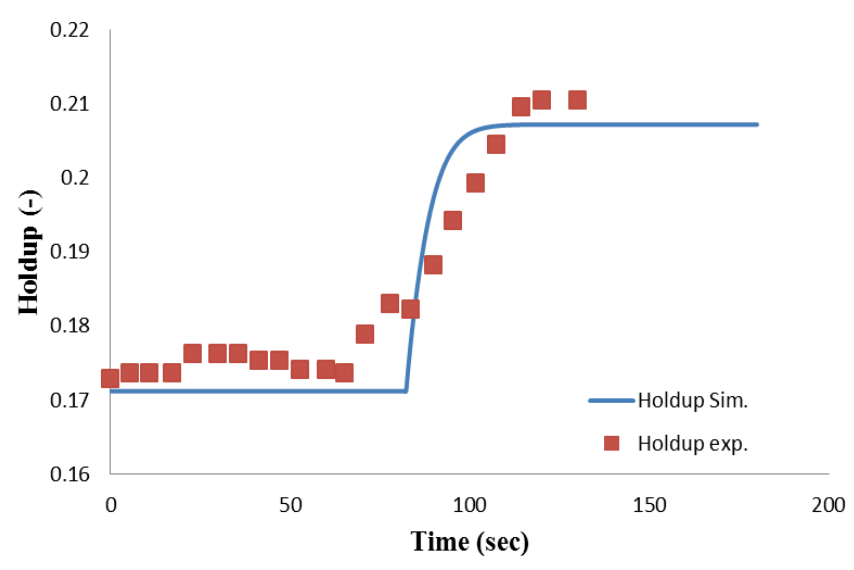

Fig. (35). Dynamic evolution of holdup profile in pulsed sieve plate column compared to the experimental data [69]. 
Table 12. Physical Properties of the Test System (w-MIBK) [70]

\begin{tabular}{|c|c|l|l|l|}
\hline \multicolumn{1}{|c|}{$\rho_{\mathrm{x}}\left(\mathrm{kg} \cdot \mathrm{m}^{-3}\right)$} & \multicolumn{1}{|c|}{$\boldsymbol{\rho}_{\mathrm{y}}\left(\mathrm{kg} \cdot \mathrm{m}^{-3}\right)$} & \multicolumn{1}{c|}{$\boldsymbol{\mu}_{\mathrm{x}}\left(\mathrm{kg} \cdot \mathrm{m}^{-1} \cdot \mathrm{sec}^{-1}\right)$} & $\mu_{\mathrm{y}}\left(\mathrm{kg} \cdot \mathrm{m}^{-1} \cdot \mathrm{sec}^{-1}\right)$ & $\sigma\left(\mathrm{N} \cdot \mathrm{m}^{-1}\right)$ \\
\hline \hline 990.9 & 800.5 & 0.001163 & 0.000554 & 0.001 \\
\hline
\end{tabular}

Table 13. Operating Conditions for Pulsed Sieve Plate Column (Dynamic Simulation)

\begin{tabular}{|c|l|l|l|l|}
\hline \multicolumn{1}{|c|}{$\mathbf{Q}_{\mathbf{c}}(\mathbf{l} / \mathbf{h})$} & \multicolumn{1}{|c|}{$\mathbf{Q}_{\mathbf{d}}(\mathbf{l} / \mathbf{h})$} & \multicolumn{1}{|c|}{ Pulse Amplitude (m) } & \multicolumn{1}{|c|}{ Pulse Frequency (s) } & No Mass Transfer \\
\hline \hline 18.36 & 17.43 & 0.032 & 1.67 & \\
\hline
\end{tabular}

Table 14. Pulsed Sieve plate Column Geometry (Dynamic Simulation)

\begin{tabular}{|l|l|}
\hline \multicolumn{1}{|c|}{ Dimension } & \multicolumn{1}{c|}{ Value } \\
\hline \hline Height of the column & $1.84(\mathrm{~m})$ \\
\hline Inlet for the continuous phase & $1.58(\mathrm{~m})$ \\
\hline Inlet for the dispersed phase & $0.35(\mathrm{~m})$ \\
\hline Column diameter & $0.051(\mathrm{~m})$ \\
\hline Diameter of sieve tray $\left(d_{s t}\right)$ & $50(\mathrm{~mm})$ \\
\hline Diameter of holes $\left(d_{h}\right)$ & $3.2(\mathrm{~mm})$ \\
\hline Relative free cross-sectional area $\left(\varphi_{s t}\right)$ & $0.23\left(\mathrm{~m}^{2} \cdot \mathrm{m}^{-2}\right)$ \\
\hline plate spacing $\left(h_{s t}\right)$ & $56(\mathrm{~mm})$ \\
\hline Number of sieve plates & $22(-)$ \\
\hline
\end{tabular}

\section{ACKNOWLEDGEMENT}

The authors acknowledge the DFG (Deutsche Forschungsgemeinschaft) - Bonn, DAAD (Deutscher Akademischer Austauschdienst) - Bonn, BASF SE - Ludwigshafen and the Federal State Research "Centre of Mathematical and Computational Modeling', - TU Kaiserslautern for the financial support and Mrs. H. Jildeh for helping in editing the manuscript.

\section{NOMENCLATURE}

$\begin{array}{ll}a & \text { Pulsation amplitude } \\ a f & \text { Pulsation intensity } \\ a_{i} & \text { Adjustable parameters } \\ a_{P K} & \text { Volumetric surface area of a packing } \\ A_{c}, A_{c o l} & \text { Column cross sectional area } \\ b_{s} & \text { Packing bridge width } \\ B & \text { Enhancement factor } \\ B_{u n} & \text { Amplifier factor } \\ C_{i} & \text { Constant parameter } \\ C_{I P} & \text { Interface instability parameter } \\ c_{c}, c_{d} & \text { Solute concentration (continuous and } \\ & \text { dispersed phase) }\end{array}$

$\begin{array}{ll}\mathrm{m} & d_{100} \\ \mathrm{~m} \cdot \mathrm{s}^{-1} & d_{c r i t} \\ \mathrm{~m}^{2} \cdot \mathrm{m}^{-3} & d_{\max } \\ \mathrm{m}^{2} & d_{s t a b} \\ \mathrm{~m} & \bar{d} \\ - & \\ - & D_{e f f} \\ - & D_{d} \\ - & f \\ \mathrm{~kg} \cdot \mathrm{m}^{-3} & g \\ & h_{p k}, h_{p k} \\ & h_{s}\end{array}$

Axial dispersion (continuous and dis- $\quad \mathrm{m}^{2} \cdot \mathrm{s}^{-1}$ persed phase)

$d_{h} \quad$ Diameter of sieve plates' holes $\quad \mathrm{m}$

$d_{L} \quad$ Hole diameter $\mathrm{m}$

$d_{T T} \quad$ Daughter droplet diameter $\quad \mathrm{m}$

$d, d^{\prime}, d_{m} \quad$ Droplet diameter m

$d_{32} \quad$ Sauter diameter $\quad \mathrm{m}$

Characteristic droplet diameter due to $\mathrm{m}$ $100 \%$ breakage probability

Critical droplet diameter

Maximum droplet diameter

Stable droplet diameter

Average linear diameter of the droplet $\mathrm{m}$ spectrum

Effective diffusion coefficient

Diffusion coefficient

Pulsation frequency

Gravitational constant

Height of packing element

Subsequent height

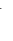

(n)

$\mathrm{m}$

$\mathrm{m}$

$\mathrm{m}$

$\mathrm{m}$

$\mathrm{m}$

$\mathrm{m}^{2} \cdot \mathrm{s}^{-2}$

$\mathrm{m}^{2} \cdot \mathrm{s}^{-2}$

$\mathrm{s}^{-1}$

$\mathrm{m} \cdot \mathrm{s}^{-2}$

$\mathrm{m}$

$\mathrm{m}$ 


\begin{tabular}{|c|c|c|c|c|}
\hline$\Delta h_{T}$ & Droplet path through the packing & $\mathrm{m}$ & $z_{d}, z_{y}$ & Dispersed feed inlet \\
\hline$H$ & Column and single compartment heights & $\mathrm{m}$ & \multirow{2}{*}{\multicolumn{2}{|c|}{ GREEK SYMBOLS }} \\
\hline$H_{c d}$ & Hamaker coefficient & $\mathrm{Nm}$ & & \\
\hline$k_{v}$ & Slowing factor & - & $\Gamma$ & Droplet breakage frequency \\
\hline$k_{c}, k_{d}$ & $\begin{array}{l}\text { Individual mass transfer coefficient (con- } \\
\text { tinuous and dispersed phase) }\end{array}$ & $\mathrm{m} \cdot \mathrm{s}^{-1}$ & $\Delta t$ & $\begin{array}{l}\text { Time interval } \\
\text { Energy dissipation }\end{array}$ \\
\hline$K_{o c}, K_{o d}$ & Overall mass transfer coefficient (con- & $\mathrm{m} \cdot \mathrm{s}^{-1}$ & $\xi_{i}$ & Adjusted parameter \\
\hline & & & $\mu_{c,} \mu_{d}$ & Viscosity (continuous and dispersed phase) \\
\hline$M$ & Variable exponent & - & $\rho_{c}, \rho_{d}$ & Density (continuous and dispersed phase) \\
\hline$m_{i}$ & Distribution coefficient & $\mathrm{kg} \cdot \mathrm{kg}^{-1}$ & $\sigma$ & Interfacial tension \\
\hline$n_{f}$ & Normalized droplets number distribution & - & $v, v^{\prime}$ & Droplet volumes \\
\hline & 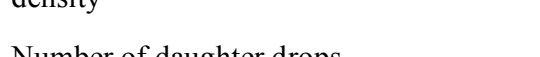 & & $v_{\times}$ & Droplet terminal velocity \\
\hline$N_{z}$ & Number of daughter drops & - & $\phi$ & Holdup \\
\hline $\bar{N}_{z}$ & Average number of daughter drops & - & $\Phi$ & Relative opening free area \\
\hline$N_{L U K}$ & Number of the droplets dispersion & - & $\vartheta\left(d^{\prime}\right)$ & Mean number of daughter droplets \\
\hline$P_{S}, P_{B}$ & Breakage probability & - & $\omega$ & Coalescence frequency \\
\hline$P_{K}, P_{C}$ & Coalescence probability & - & $\Omega$ & Mixing parameter \\
\hline$Q_{c}, Q_{d}$ & $\begin{array}{l}\text { Volumetric flow rate (continuous and } \\
\text { dispersed phase) }\end{array}$ & $\mathrm{m}^{3} \cdot \mathrm{s}^{-1}$ & $\tau_{k}$ & Droplet average life \\
\hline
\end{tabular}

\section{DIMENSIONLESS NUMBERS}

$t \quad$ Time s

$\begin{array}{ll}V_{a b s} & \text { Absolute velocity } \\ V_{c}, V_{d} & \begin{array}{l}\text { Relative velocity (continuous and dis- } \\ \text { persed phase) }\end{array}\end{array}$

$\begin{array}{ll}V_{c h a r} & \text { Characteristic velocity } \\ V_{\max } & \text { Maximum velocity } \\ V_{r} & \text { Relative droplet (slip) velocity } \\ V_{r}^{*}(d) & \begin{array}{l}\text { Relative velocity of a single droplet in } \\ \text { the column }\end{array}\end{array}$

$\begin{array}{ll}V_{t} & \text { Terminal droplet velocity } \\ v_{d} & \text { Drift by convection } \\ v(d) & \text { Volume of a droplet of diameter } d\end{array}$

$v(d) \quad$ Volume of a droplet of diameter $d \quad \mathrm{~m}$

$v_{\min }, v_{\max } \quad$ Maximum and minimum droplet volume $\mathrm{m}^{3}$ of diameter $d$

\begin{tabular}{|c|c|c|}
\hline$x$ & $\begin{array}{l}\text { Solute concentration for continuous } \\
\text { phase }\end{array}$ & $\mathrm{kg} \cdot \mathrm{m}^{-3}$ \\
\hline$e^{*}$ & $\begin{array}{l}\text { Solute concentration for continuous } \\
\text { phase at equilibrium }\end{array}$ & $\mathrm{kg} \cdot \mathrm{m}^{-3}$ \\
\hline$y$ & Solute concentration for dispersed phase & kg. $\mathrm{m}^{-3}$ \\
\hline$y^{*}$ & $\begin{array}{l}\text { Solute concentration for dispersed phase } \\
\text { at equilibrium }\end{array}$ & $\mathrm{kg} \cdot \mathrm{m}^{-3}$ \\
\hline & Spatial coordinate & \\
\hline
\end{tabular}

$A_{r} \quad$ Archimedes number

$\mathrm{m} . \mathrm{s}^{-1} \quad E \ddot{O} \quad$ Eötvös number

$\mathrm{m} . \mathrm{s}^{-1}$

$\mathrm{m} \cdot \mathrm{s}^{-1}$

$F o_{d} \quad$ Fourier number of the dispersed phase

$\mathrm{m} . \mathrm{s}^{-1} \quad$ Re Reynolds number

m.s. $\quad \pi_{a f} \quad$ Pulsation intensity

m.s s $^{-1} \quad \pi_{a p} \quad$ Volumetric surface area of a packing

$\pi_{d}-1-\pi^{-1}-$ Droplet diameter

m.s $^{-1} \quad \pi_{h p} \quad$ Height of a packing

$\mathrm{m} . \mathrm{s}^{-1} \quad \pi_{\sigma} \quad$ Interfacial tension

$\mathrm{s}^{-1}$
$\mathrm{~s}$
$\mathrm{~m}^{2} \cdot \mathrm{s}^{-3}$
-
$\mathrm{kg} \cdot \mathrm{m}^{-1} \cdot \mathrm{s}^{-1}$
$\mathrm{~kg} \cdot \mathrm{m}^{-3}$
$\mathrm{~N} \cdot \mathrm{m}^{-1}$
$\mathrm{~m}^{3}$
$\mathrm{~m} \cdot \mathrm{s}^{-1}$
-
-
-
$\mathrm{m}^{3} \cdot \mathrm{s}^{-1}$
-
$\mathrm{s}$

\section{REFERENCES}

[1] T.C. Lo, M.H.I. Bair, and C. Hanson, Handbook of solvent extraction. New York: John Wiley \& Sons, 1983.

[2] H.-J. Bart, Reactive Extraction. Berlin: Springer, 2001.

[3] G.S. Luo, H.B. Li, X.J. Tang, and J.D. Wang, "Drop breakage in a coalescence-dispersion pulsed-sieve-plate extraction column", Chem. Eng. J., vol. 102, No. 2, pp. 185-191, Sept 2004.

[4] M. M. Attarakih, H.-J. Bart, and N.M. Faqir, "Numerical Solution of Bivariate Population Balance Equation for the Interacting Hydrodynamics and Mass Transfer in Liquid-Liquid Extraction Columns," Chem. Eng. Sci., vol. 61, pp. 113-123, Jan 2006.

[5] M.M. Attarakih, H.-J Bart, G.L. Lagar and N.M. Faqir, "LLECMOD: A Window-based program for hydrodynamics simulation of liquid-liquid extraction columns," Chem. Eng. Proc., vol. 45, pp. 113-123, Feb 2006.

[6] M.M. Attarakih, H.-J. Bart, T. Steinmetz, M. Dietzen, and N.M. Faqir, "LLECMOD: A bivariate population Balance simulation tool 
for liquid-liquid extraction columns", Open Chem. Eng. J., vol. 2, pp. 10-34, 2008.

[7] M. Jaradat, M. Attarakih, and H.-J. Bart, "Effect of phase dispersion and mass transfer direction on steady state RDC performance using population balance modelling", Chem. Eng. J., vol. 165, No. 2, pp. 379-387, Dec 2010.

[8] H. Hufnagl, M. McIntyre, and E. Blaß, "Dynamic behaviour and simulation of a liquid-liquid extraction column", Chem. Eng. Technol., vol. 14, pp. 301-306, Oct 1991.

[9] O. Weinstein, R. Semiat and D. Lewin, "Modelling, simulation and control of liquid-liquid extraction columns", Chem. Eng. Sci., vol. 53, pp. 325-339, Jan 1998.

[10] F.S. Mjalli, N.M. Abdel-Jabbar, and J.P. Fletcher, "Modelling, simulation and control of a scheibel liquid-liquid contactor: Part 1. Dynamic analysis and system identification", Chem. Eng. Process., vol. 44, pp. 541-553, May 2005.

[11] T. Xiaojin, L. Guangsheng, and W. Jiading, "An improved dynamic combined model for evaluating the mass transfer performances in extraction columns", Chem. Eng. Sci., vol. 60, pp. 44094421, Aug 2005.

[12] E. Blass, and H. Zimmerman, "Mathematische Simulation und Experimentelle Bestimmung des Instationären Verhaltens einer Flüssigkeitspulsierten Siebbodenkolonne zur Flüssig-FlüssigExtraktion", Verfahrenstechnik, vol. 16, No. 9, pp. 652-690, 1982.

[13] J. Ingham, I.J. Dunn, E. Heinzle, J.E. Prenosil, and J.B. Snape, Chemical engineering dynamics: An introduction to modelling and computer simulation. Weinheim: Wiley-VCH Verlag GmbH \& Co. KgaA, 2007.

[14] M.M. Attarakih, D. Zeidan, C. Drumm, S. Tiwari, J. Kuhnert, H. Allaboun, and H.-J. Bart, "Dynamic Modelling of Liquid Extraction Columns using the Direct Primary and Secondary Particle Method (DPSPM)," In 6th International Conference on Computational Fluid Dynamics in the Oil \& Gas, Metallurgical and Process Industries, 2008.

[15] M.M. Attarakih, M. Jaradat, H. Allaboun, H.-J. Bart, and N.M. Faqir, "Dynamic modelling of a rotating disk contactor using the primary and secondary particle method (PSPM)", In 18th European Symposium on Computer Aided Process Engineering, 2008.

[16] H.-J. Bart, and G. Stevens, "Reactive solvent extraction," in Ion exchange and solvent extraction, Vol. 17, Kertes M., Sengupta A.K. (Eds.), New York: Marcel Dekker, 2004, pp. 37-82.

[17] M.O. Garg and H.R.C. Pratt, "Measurement and modelling of droplet coalescence and breakage in a pulsed-plate extraction column”, AIChE J., vol. 30, No. 3, pp. 432-441, May 1984.

[18] G. Casamatta, and A. Vogelpohl, "Modelling of fluid dynamics and mass transfer in extraction columns", Ger. Chem. Eng., vol. 8, pp. 96-103, 1985.

[19] S.D. Al Khani, C. Gourdon, and G. Casamatta, "Dynamic and steady-state simulation of hydrodynamics and mass transfer in liquid-liquid extraction column", Chem. Eng. Sci., Vol. 44, No. 6, pp. 1295-1305, 1989.

[20] J.F. Milot, J. Duhamet, C. Gourdon, and G. Casamatta, "Simulation of a pneumatically pulsed liquid-liquid extraction column", Chem. Eng. J., vol. 45, pp. 111-122, Dec 1990.

[21] C. Gourdon, G. Casamatta, and G. Muratet, "Population balance based modelling of solvent extraction columns", in Liquid-liquid extraction equipment, J.C. Godfrey and M.J. Slater (Eds.), Chichester: Wiley, 1994, pp. 137-226.

[22] T. Kronberger, A. Ortner, W. Zulehner, and H.-J. Bart, "Numerical simulation of extraction columns using a drop population model", Comput. Chem. Eng., vol. 19, pp. 639-644, Jun 1995.

[23] M.M. Attarakih, H.J. Bart, and N.M. Faqir, "Numerical solution of the spatially distributed population balance equation describing the hydrodynamics of interacting liquid-liquid dispersions," Chem. Eng. Sci., vol. 59, pp. 2567-2592, Jun 2004.

[24] M.M. Attarakih, H.J. Bart, and N.M. Faqir, "Solution of the droplet breakage equation for interacting liquid-liquid dispersions: a conservative discretization approach," Chem. Eng. Sci., vol. 59, pp. 2547-2565, Jun 2004.

[25] M. Attarakih, M. Jaradat, C. Drumm, H.-J. Bart, S. Tiwari, V.K. Sharma, J. Kuhnert, and A. Klar, "A multivariate population balance model for liquid extraction columns", In $19^{\text {th }}$ European Symposium on Computer Aided Process Engineering, 2009.

[26] J.C. Godfrey, and M.J. Slater, Eds., Liquid-liquid extraction equipment, Chichester: J. Wiley \& Sons, 1994.
[27] T. Kronberger, "Numerische Simulation von Tropfenpopulationen in Extraktionskolonnen", PhD Thesis, Universität Linz, Linz, Austria, 1995.

[28] G. Zamponi, J. Stichlmair, A. Gerstlauer, and E.D. Gilles, "Simulation of the transient behaviour of a stirred liquid-liquid extraction column", Comput. Chem. Eng., vol. 20, pp. 963-968, 1996.

[29] D. Ramkrishna, Population balances: Theory and applications to particulate systems in engineering, Academic Press, San Diego, 2000.

[30] M.J. Goodson, "Stochastic solution of multi-dimensional population balances", PhD Thesis, Trinity College-University of Cambridge, Cambridge, United Kingdom, 2007.

[31] G. Modes, Grundsätzliche Studie zur Populationsdynamik einer Extraktionskolonne auf Basis von Einzeltropfenuntersuchungen, Aachen: Shaker Verlag, 2000.

[32] C.A. Coulaloglou, and L.L. Tavlarides, "Description of interaction processes in agitated liquid-liquid dispersions". Chem. Eng. Sci., vol. 32, No. 11, pp. 1289-1297, Oct 1977.

[33] Y. Liao, and D. Lucas, "A literature review on mechanisms and models for the coalescence process of fluid particles," Chem. Eng. Sci., vol. 65, No. 10, pp. 2851-2864, May 2010.

[34] S. Kumar, and D. Ramkrishna, "On the solution of population balance equations by discretization - I. A fixed pivot technique", Chem. Eng. Sci., vol. 51, pp. 1311-1332, Apr 1996.

[35] R. Clift, J.R. Grace, and M.E. Weber, Bubbles, drops and particles, New York: Academic Press, 1978.

[36] J.A. Wesselingh, and A.M. Bollen, "Single particles, bubbles and drops: their velocities and mass transfer coefficients", Trans. Inst. Chem. Eng., vol. 77, pp. 89-96, Mar 1999.

[37] M. Henschke, Auslegung pulsierter Siebboden-Extraktionskolonnen, Shaker Verlag Aachen, 2004.

[38] A.J. Klee, and R.E. Treybal, "Rate of rise or fall of liquid drops", AIChE J., vol. 2, No. 4, pp. 444-447, Dec 1956.

[39] A. Vignes, "Hydrodynamique des dispersions", Genie Chimique, vol. 93, pp. 129-142, 1965.

[40] J.R. Grace, T. Wairegi, and T.H. Nguyen, "Shapes and velocities of single drops and bubbles moving freely through immiscible liquids", Trans. Inst. Chem. Eng., vol. 54, pp. 167-173, Apr 1976.

[41] D. Garthe, "Fluid dynamics and mass transfer of single particles and swarms of particles in extraction column", PhD Thesis, TU München, München, Germany, 2006.

[42] R. Gayler, N. Roberts, and H. Pratt, "Liquid-liquid extraction. Part IV. A further study of holdup in packed columns", Trans. Inst. Chem. Eng., vol. 31, pp. 57-68, 1953.

[43] H. Haverland, "Untersuchungen zur Tropfendispergierung in flüssigkeitspulsierten Siebboden-Extraktionskolonnen”, PhD Thesis, Technical University Clausthal, Clausthal,Germany.

[44] G. Wagner, and E. Blass, "Modelling of drop disintegration in liquid pulsed sieve-tray extractors with regard to viscosity", Chem. Eng. Technol., vol. 21, No. 6, pp. 475-479, Jun 1999.

[45] H. Bahmanyar, and M.J. Slater, "Studies of drop breakup in liquidliquid systems in a rotary disc contactor, Part I: Conditions of no mass transfer," Chem. Eng. Technol., vol. 14, pp. 79-89, 1991.

[46] T. Leu, Beitrag zur Fluiddynamik von Extraktionskolonnen mit geordneten Packungen, VDI Verlag, Düsseldorf, 1995.

[47] S. Hartland, and D.K. Vohra, "Koaleszenz in vertikalen dichtgepackten Dispersionen", Chem. Ing. Tech., vol. 50, Nr. 9, pp. 673682, January 1978.

[48] D.V. Smith, and G.A. Davies, "Coalescence in droplet dispersions”, Can. J. Chem. Eng., vol. 48, pp. 628-637, Aug 1970.

[49] M. Hozawa, U. Suzuki, T. Tadaki, and S. Maeda, "The mechanism of formation of droplet bed at a liquid-liquid interface", Kagaku Kógaku, vol. 37, No. 4, pp. 402-408, 1973.

[50] M. Henschke, Dimensionierung liegender Flüssig-flüssigAbscheider anhand diskontinuierlicher Absetzversuche, FortschrittBerichte VDI, VDI Verlag, Düsseldorf, Germany,1995.

[51] E. Rohlfing, Untersuchungen zum fluiddynamischen Verhalten einer pulsierten Füllkörperextraktionskolonne, Fortschritt-Berichte VDI, VDI Verlag, Düsseldorf, Germany,1992.

[52] S. Klinger, "Messung und Modellierung des Spaltungs- und Koaleszenzverhaltens von Tropfen bei der Extraktion", PhD Thesis, RWTH Aachen, Aachen, Germany, 2008.

[53] A.E. Handlos, and T. Baron, "Mass and heat transfer from drops in liquid-liquid extraction", AIChE J., vol. 3, No. 1, pp. 127-136, Mar 1957. 
[54] A. Kumar, and S. Hartland, "Correlations for prediction of mass transfer coefficients in single drop systems and liquid-liquid extraction columns", Trans. Inst. Chem. Eng., vol. 77, pp. 372-384, Jul 1999.

[55] A.B. Newman, "The drying of porous solids: Diffusion and surface emission equations", Trans. Am. Inst. Chem. Eng., vol. 27, pp. 203$220,1931$.

[56] R. Kronig, and J.C. Brink, "On the theory of extraction from falling droplets", Appl. Sci. Res., vol. 2, No. 1, pp. 142-154, Mar 1950.

[57] M. Henschke, and A. Pfennig, "Mass-transfer enhancement in single-drop extraction experiments", AIChE J., vol. 45, No. 10, pp. 2079-2086, Oct 1999.

[58] M. Laso, L. Steiner, and S. Hartland, "Dynamic simulation of agitated liquid-liquid dispersions-II. Experimental determination of breakage and coalescence rates in a stirred tank", Chem. Eng. Sci., vol. 42, No. 10, pp. 2437-2445, 1987.

[59] G. Narsimhan, G. Nejfelt, and D. Ramkrishna, "Breakage functions for droplets in agitated liquid-liquid dispersion," AIChE J., vol. 30, No. 3, pp. 457-467, May 1984.

[60] S.A. Schmidt, M. Simon, and H.-J. Bart "Tropfenpopulationsmodellierung Einfluss von Stoffsystem und technischen Geometrien", Chem. Eng. Technol., vol. 75, No. 1-2, pp. 62-68, Feb 2003.

[61] H. Sovova, "Breakage and coalescence of drops in a batch stirred vessel-II. Comparison of model and experiments", Chem. Eng. Sci., vol. 36, No. 9, pp. 1567-1573, Jan 1981.

[62] C. Tsouris, and L.L. Tavlarides, "Breakage and coalescence models for drops in turbulent dispersions", AIChE J., vol. 40, No. 3, pp. 395-406, Mar 1994.
[63] R. Bauer, "Die Längsvermischung beider Phasen in einer gerührten Fest-Flüssig-Extraktionskolonne", PhD Thesis, ETH Zurich, Zurich, Switzerland, 1976.

[64] W.J. Korschinsky and S. Azimzadeh-Khatayloo, "An improved stagewise model of countercurrent flow liquid-liquid contactors", Chem. Eng. Sci., vol. 31, No. 10, pp. 871-875, Jan 1976.

[65] B.H. Wolschner, "Konzentrationsprofile in Drehscheibenextraktoren", PhD Thesis, Technische Universität Graz, Graz, Austria, 1980.

[66] A. Kumar, and S. Hartland, "Mass transfer in a Kühni column", Ind. Eng. Chem. Res., vol. 27, pp. 1198-1203, Jul 1988.

[67] M.J. Slater, "A combined model of mass transfer coefficients for contaminated drop liquid-liquid systems", Can. J. Chem. Eng., vol. 73, No. 4, pp. 462-469, Aug 1995.

[68] M.J. Slater, "Rate coefficients in liquid-liquid extraction studies", in Liquid-liquid extraction equipment, J.C. Godfrey and J.M. Slater, (Eds.), Chichester: John Wiley \& Sons, 1994.

[69] H.R. Foster Jr., R.E. McKEE, and A.L. Babb, "Transient holdup behaviour of a pulsed sieve plate solvent extraction column", Ind. Eng. Chem. Process Des. Dev., vol. 9, No. 2, pp. 272-278, Apr 1970.

[70] G.A. Sehmel, and A.L. Babb, "Holdup studies in a pulsed sieveplate solvent extraction column", Ind. Eng. Chem. Process Des. Dev., vol. 2, No. 1, pp. 38-42, Jan 1963.

(C) Jaradat et al.; Licensee Bentham Open.

This is an open access article licensed under the terms of the Creative Commons Attribution Non-Commercial License (http://creativecommons.org/licenses/by-nc/3.0/) which permits unrestricted, non-commercial use, distribution and reproduction in any medium, provided the work is properly cited. 